\title{
Spin-wave spectrum of a two-dimensional itinerant electron system: Analytic results for the incommensurate spiral phase in the strong-coupling limit
}

\author{
E. Arrigoni \\ Institut für Theoretische Physik, Universität Würzburg \\ D-97074 Würzburg, Germany \\ G.C. Strinati \\ Dipartimento di Matematica e Fisica, Sezione INFM \\ Università di Camerino, I-62032 Camerino (MC), Italy
}

(November 5, 2018)

\begin{abstract}
We study the zero-temperature spin fluctuations of a two-dimensional itinerant-electron system with an incommensurate magnetic ground state described by a single-band Hubbard Hamiltonian. We introduce the (broken-symmetry) magnetic phase at the mean-field (Hartree-Fock) level through a spiral spin configuration with characteristic wave vector $\mathbf{Q}$ different in general from the antiferromagnetic wave vector $\mathbf{Q}_{\mathbf{A F}}$, and consider spin fluctuations over and above it within the electronic random-phase (RPA) approximation. We obtain a closed system of equations for the generalized wave vector and frequency dependent susceptibilities, which are equivalent to the ones reported recently by Brenig. We obtain, in addition, analytic results for the spin-wave dispersion relation in the strong-coupling limit of the Hubbard Hamiltonian and find that at finite doping the spin-wave dispersion relation has a hybrid form between that associated with the (localized) Heisenberg model and that associated with the (long-range) RKKY exchange interaction. We also find an instability of the spin-wave spectrum in a finite region about the center of the Brillouin zone, which signals a physical instability toward a different spin- or, possibly, charge-ordered phase, as, for example, the stripe structures observed in the high-Tc materials. We expect, however, on physical grounds that for wave vectors external to this region the spin-wave spectrum that we have determined should survive consideration of more sophisticated mean-field solutions.
\end{abstract}

\section{INTRODUCTION}

Spin fluctuations about incommensurate static magnetic configurations represent an interesting problem, especially in the view of the accumulating experimental evidence on the parent compounds of the high- $T_{c}$ cuprate materials.t The dynamic structure factor $S(\mathbf{q}, \omega)$ at wave vector $\mathbf{q}$ and frequency $\omega$, as measured by inelastic neutron scattering, shows noticeable peaks over the background when $\mathbf{q}=\mathbf{Q}=\mathbf{Q}_{\mathbf{A F}}+\Delta \mathbf{Q}\left(|\Delta \mathbf{Q}| \ll\left|\mathbf{Q}_{\mathbf{A F}}\right|\right.$ for increasing dopant concentration, at finite albeit small values of $\omega$ (with $\omega \ll J, J$ being the exchange coupling). studies for larger values of $\left|\mathbf{q}-\mathbf{Q}_{\mathbf{A F}}\right|$ have also detected the presence of well-defined spin-wave-type excitations close to the boundary of the antiferromagnetic (AF) Brillouin zone (BZ). The fact that spin-wave excitations with small wavelength (i.e., comparable to lattice spacing) can be detected even in the absence of long-range magnetic order, has actually been well established experimentally since the eighties also in more conventional magnetic materials.t This state of affairs has, in thurn, prompted a number of theoretical studies on dynamical excitations about incommensurate spin configurations. 5 目

From the theoretical point of view, inclusion of incommensurate spin configurations in cluster mean-field calculations would require one to consider cluster sizes at least as big as the spatial extension of the incommensurability. In this way, complex incommensurate patterns could as well be included in the calculation, at the price of considerable numerical effort and without full analytic control on the results. Alternatively, one may set up calculations for an infinite system with necessarily simpler incommensurate spin configurations, with the advantage, however, of obtaining analytic results (at least) in some limits, which in turn may admit a simple physical interpretation. In this respect, the spiral spin configuration appears to be the only one for which analytic calculations can be performed, in the sense that the constituent equations can be brought to a closed form which is manageable for controlled analytic approximations. Indeed previous analytic calculations have considered statifitong-range background spiral spin configurations, on top of which dynamical spin excitations have been considered.

The intrinsic simplicity of the background spiral spin configuration and the neglect of its dynamics have resulted, however, into an instability of the spin-wave spectrum in a limited region of the Brillouin zone This outcome could, in principle, make the resulting spin-wave spectrum altogether unreliable, as the underlying spin configuration (over and above which spin-wave excitations are constructed) could be much more complex than a spiral one and even possess a dynamics of its own. In addition, a static or dynamic charge modulation can be present as well, like the stripe structures observed in some underdoped cuprates 9 One expects, however, on physical grounds the short- 
wavelength spin-wave excitations, obtained on top of a spiral configuration, to preserve their dispersion relation even when considering more complex long-range underlying structures.

Within this framework, we have pursued the analytic calculation with an underlying spiral spin configuration for an itinerant electronic system described by a single-band Hubbard Hamiltonian. The spin (and charge) dynamics have bee described within the electronic random-phase (RPA) approximation for the dynamical susceptibilities, based on a broken-symmetry Hartree-Fock (HF) mean-field solution, along the lines of Ref I. In particular, we have considered the large $U / t$ limit in detail (where $t$ is the nearest-neighbor hopping matrix element and $U$ is the local on-site repulsion in the Hubbard Hamiltonian), for which the spin-wave dispersion relation (as obtained from the poles of the dynamical susceptibilities) can be expressed in analytic form by systematically expanding in the small parameter $t / U$. In this way, we have obtained an analytic expression for the spin-wave dispersion relation valid in the limit $t / U \ll 1$ [cf. Eq. (57) below], showing a novel characteristic structure, namely, a hyprid form between the dispersion relations obtained within the nearest-neighbor Heisenberg model for localized spin迆 and the long-range RKKY interaction mediated by the conduction electrons.11

This analytic form of the dispersion relation could admittedly not have been guessed a priori, by fitting the dispersion relation obtained numerically (from the location of the poles of the dynamical susceptibilities) with a Heisenberg model extending in principle to a large albeit finite number of neighbors. Neither, this analytic form can be simply reduced to a nearest-neighbor Heisenberg dispersion relation with a doping-dependent exchange integral.12 Rather, the characteristic long-range RKKY contribution would require fitting to a Heisenberg model with an infinite number of neighbors. This would contradict the spirit with which the Heisenberg model was introduced to start with, 13 namely, as a fitting model that makes physical sense when the interactions extend to a limited number of neighbors only. Note that this situation contrasts that found at half-filling of the Hubbard band (i.e., in the absence of doping), where the antiferromagnetic (AF) spin-wave spectrum can be nicely fitted by a Heisenberg model extending at most to a few neighbors 14 Our results also show that the magnitude of the overall exchange integral, which characterizes the spin-wave spectrum, decreases with increasing doping and vanishes when the transition to a ferromagnetic case occurs at the mean-field level.

The plan of the paper is as follows. Section 2 obtains the implicit form of the spin-wave dispersion relation within the HF-RPA approximations, by solving for the dynamical susceptibilities of the itinerant electron system in the presence of an incommensurate spin spiral ground state. Section 3 focuses on the small $t / U$ expansion of the results of Section 2, which requires a careful analysis of the doping dependence of the relevant quantities. Section 4 discusses the main results of this paper and Section 5 gives our conclusions. For the sake of completeness, we report in the Appendices details of the analytic calculations, and adapt know results for the Heisenberg and RKKY spin-wave spectra to the present context.

\section{DYNAMICAL SUSCEPTIBILITIES WITHIN THE ITINERANT-ELECTRON RPA APPROXIMATION WITH AN INCOMMENSURATE SPIN-SPIRAL GROUND STATE}

In this Section, we give the derivation of the spin-wave dispersion relation for a two-dimensional itinerant-electron system in the presence of an incommensurate spiral magnetic structure with a generic characteristic wave vector $\mathbf{Q}$, within the electronic RPA approximation. Although our results for the dispersion relation coincide with those previously given by Brenig in Ref.U, we provide here in addition the expression of the correlation functions which can be relevant for a direct comparison with neutron scattering experiments. Further details of the calculation are reported in Appendix A.

We emphasize that the finding of a closed-form expression for the correlation functions for any characteristic wave vector $\mathbf{Q}$ (incommensurate with the lattice spacing) and not just for the (commensurate) antiferromagnetic wave vector $\mathbf{Q}_{A F}$ is altogether a nontrivial result, being intrinsically related to the peculiar pattern of the spiral magnetic solution for the ground state.

We begin by considering the generalized correlation function at zero temperature in the broken-symmetry phase:

$$
\mathcal{X}_{\mu, \nu}\left(\mathbf{r} t, \mathbf{r}^{\prime} t^{\prime}\right)=-i\left\langle T\left[S_{\mu}(\mathbf{r}, t) S_{\nu}\left(\mathbf{r}^{\prime}, t^{\prime}\right)\right]\right\rangle+i\left\langle S_{\mu}(\mathbf{r}, t)\right\rangle\left\langle S_{\nu}\left(\mathbf{r}^{\prime}, t^{\prime}\right)\right\rangle
$$

where the average $\langle\cdots\rangle$ is taken over the ground state, $T$ stands here for Wick's time-ordering operator, and $S_{\mu}(\mathbf{r})$ is given by (we set $\hbar=1$ throughout) 


$$
S_{\mu}(\mathbf{r})=\frac{1}{2} \sum_{\alpha, \beta} \psi_{\alpha}^{\dagger}(\mathbf{r}) \sigma_{\alpha, \beta}^{\mu} \psi_{\beta}(\mathbf{r})
$$

In these expressions, $\mu, \nu=(0, x, y, z), \sigma^{\mu}$ is a Pauli matrix (with $\sigma^{0}$ equal to the $2 \times 2$ identity matrix), and $\alpha, \beta$ are spin labels. Note that $2 S_{0}(\mathbf{r})$ coincides with the density operator, which couples with the spin operator in the presence of an incommensurate spiral magnetic structure.

For the simple band we are considering, the field operator in Eq.(2) can be represented in the form

$$
\psi_{\alpha}(\mathbf{r})=\sum_{i} \phi\left(\mathbf{r}-\mathbf{R}_{i}\right) c_{i \alpha}
$$

where $\phi(\mathbf{r})$ is the atomic (Wannier) orbital associated with the simple band, $\mathbf{R}_{i}$ is the lattice vector locating site $i$, and $c_{i \alpha}$ is a destruction operator. Time evolution in Eq. (1D) is governed by the Heisenberg representation:

$$
\psi_{\alpha}(\mathbf{r}, t)=\mathrm{e}^{i H t} \psi_{\alpha}(\mathbf{r}) \mathrm{e}^{-i H t}
$$

where for $H$ we take the simple-band two-dimensionalHubbard Hamiltonian.

In terms of the two-particle correlation function $L, 15$ the generalized correlation function (11) takes the form

$$
\mathcal{X}_{\mu, \nu}\left(\mathbf{r} t, \mathbf{r}^{\prime} t^{\prime}\right)=-\frac{i}{4} \sum_{\alpha, \beta} \sum_{\alpha^{\prime}, \beta^{\prime}} \sigma_{\alpha, \beta}^{\mu} \sigma_{\alpha^{\prime}, \beta^{\prime}}^{\nu} L\left(\mathbf{r} t \beta, \mathbf{r}^{\prime} t^{\prime} \beta^{\prime} ; \mathbf{r} t^{+} \alpha, \mathbf{r}^{\prime} t^{\prime+} \alpha^{\prime}\right)
$$

with $t^{+}=t+\eta\left(\eta=0^{+}\right)$, where $L$ satisfies the Bethe-Salpeter equation:

$$
\begin{aligned}
L\left(1,2 ; 1^{\prime}, 2^{\prime}\right) & =G\left(1,2^{\prime}\right) G\left(2,1^{\prime}\right) \\
& +\int d 3 d 4 d 5 d 6 G(1,3) G\left(4,1^{\prime}\right) \Xi(3,5 ; 4,6) L\left(6,2 ; 5,2^{\prime}\right)
\end{aligned}
$$

$(1,2, \cdots$ signifying the set of space, spin, and time variables). In the above expression,

$$
G(1,2)=-i\left\langle T\left[\psi(1) \psi^{\dagger}(2)\right]\right\rangle
$$

is the single-particle Green's function and the kernel $\Xi$ represents an effective two-particle interaction. In particular, within the RPA approximation we are adopting, the kernel $\Xi$ takes the form:

$$
\begin{aligned}
\Xi(3,5 ; 4,6) & =-i v_{0} \delta(3,4) \delta\left(5,6^{+}\right) \delta\left(x_{3}, x_{6}\right) \delta\left(\alpha_{3},-\alpha_{6}\right) \\
& +i v_{0} \delta(3,6) \delta(4,5) \delta\left(x_{3}^{+}, x_{4}\right) \delta\left(\alpha_{3},-\alpha_{4}\right)
\end{aligned}
$$

with the notation $x \equiv(\mathbf{r}, t)$ and where the constant $v_{0}$ can be related to the parameter $U$ of the Hubbard Hamiltonian as follows:

$$
U=v_{0} \int d \mathbf{r}|\phi(\mathbf{r})|^{4}
$$

Entering Eqs.(6) and (8) into Eq.(5), we obtain for the generalized correlation function within the RPA approximation:

$$
\begin{aligned}
\mathcal{X}_{\mu, \nu}\left(x, x^{\prime}\right) & =-\frac{i}{4} \sum_{\alpha, \beta} \sum_{\alpha^{\prime}, \beta^{\prime}} \sigma_{\alpha, \beta}^{\mu} \sigma_{\alpha^{\prime}, \beta^{\prime}}^{\nu} G\left(x \beta, x^{\prime} \alpha^{\prime}\right) G\left(x^{\prime} \beta^{\prime}, x \alpha\right) \\
& +\frac{v_{0}}{4}(-i)^{2} \sum_{\alpha, \beta} \sum_{\alpha^{\prime}, \beta^{\prime}} \sigma_{\alpha, \beta}^{\mu} \sigma_{\alpha^{\prime}, \beta^{\prime}}^{\nu} \int d 3 G\left(x \beta, x_{3} \alpha_{3}\right) G\left(x_{3} \alpha_{3}, x \alpha\right) \\
& \times L\left(x_{3} \bar{\alpha}_{3}, x^{\prime} \beta^{\prime} ; x_{3}^{+} \overline{\alpha_{3}}, x^{\prime+} \alpha^{\prime}\right) \\
& -\frac{v_{0}}{4}(-i)^{2} \sum_{\alpha, \beta} \sum_{\alpha^{\prime}, \beta^{\prime}} \sigma_{\alpha, \beta}^{\mu} \sigma_{\alpha^{\prime}, \beta^{\prime}}^{\nu} \int d 3 G\left(x \beta, x_{3} \alpha_{3}\right) G\left(x_{3} \overline{\alpha_{3}}, x \alpha\right) \\
& \times L\left(x_{3} \alpha_{3}, x^{\prime} \beta^{\prime} ; x_{3}^{+} \overline{\alpha_{3}}, x^{\prime+} \alpha^{\prime}\right)
\end{aligned}
$$

with $\bar{\alpha}=-\alpha$. This is apparently not a closed-form equation for $\mathcal{X}$ itself. By the manipulations reported in Appendix A, however, Eq. (10) can be cast in the form of a coupled set of equations for the matrix components of the correlation function, as follows: 


$$
\mathcal{X}_{\mu, \nu}\left(x, x^{\prime}\right)=\mathcal{X}_{\mu, \nu}^{(0)}\left(x, x^{\prime}\right)+2 v_{0} \sum_{\mu^{\prime}, \nu^{\prime}} \int d x^{\prime \prime} \mathcal{X}_{\mu, \mu^{\prime}}^{(0)}\left(x, x^{\prime \prime}\right) \epsilon_{\mu^{\prime}, \nu^{\prime}} \mathcal{X}_{\nu^{\prime}, \nu}\left(x^{\prime \prime}, x^{\prime}\right)
$$

where we have introduced the tensor

$$
\epsilon_{\mu, \nu}=\left(\begin{array}{rrrr}
1 & 0 & 0 & 0 \\
0 & -1 & 0 & 0 \\
0 & 0 & -1 & 0 \\
0 & 0 & 0 & -1
\end{array}\right)
$$

To solve Eq. (11), the explicit form of the non-interacting counterpart $\mathcal{X}^{(0)}$ of $\mathcal{X}$ is required. To this end, the ground-state average in Eq. (7) is evaluated as shown in Appendix A, in terms of the eigenvalues $\left(\epsilon_{r}\right)$ and eigenvectors $\left(W_{\xi, r}\right)$ of the mean-field Hubbard Hamiltonian (see also Section 3), yielding for the time Fourier transform of $\mathcal{X}^{(0)}$ the expression

$$
\begin{aligned}
\mathcal{X}_{\mu, \nu}^{(0)}\left(\mathbf{r}, \mathbf{r}^{\prime} ; \omega\right) & =\frac{1}{4 \mathcal{N}^{2}} \sum_{i, j} \sum_{\mathbf{k}, \mathbf{k}^{\prime}}^{B Z} \mathrm{e}^{i\left(\mathbf{k}-\mathbf{k}^{\prime}\right) \cdot\left(\mathbf{R}_{i}-\mathbf{R}_{j}\right)}\left|\phi\left(\mathbf{r}-\mathbf{R}_{i}\right)\right|^{2}\left|\phi\left(\mathbf{r}^{\prime}-\mathbf{R}_{j}\right)\right|^{2} \\
& \times \sum_{r, r^{\prime}} \sum_{\mu^{\prime}, \nu^{\prime}} T_{\mu, \mu^{\prime}}\left(\Omega_{i}\right) T_{\nu, \nu^{\prime}}\left(\Omega_{j}\right) F_{r^{\prime}, r}^{\mu^{\prime}}\left(\mathbf{k}^{\prime}, \mathbf{k}\right) F_{r, r^{\prime}}^{\nu^{\prime}}\left(\mathbf{k}, \mathbf{k}^{\prime}\right) \\
& \times \mathcal{F}_{r, r^{\prime}}\left(\mathbf{k}, \mathbf{k}^{\prime}, \omega\right)
\end{aligned}
$$

where $B Z$ stands for the two-dimensional Brillouin zone, $\mathcal{N}$ is the number of lattice sites, and the quantities $T, F$, and $\mathcal{F}$ are defined in Appendix A ( $\Omega_{i}$ standing for the angles defining the local spin quantization axis). We consider further the space Fourier transform

$$
\begin{aligned}
\mathcal{X}_{a b}\left(\mathbf{q}, \mathbf{q}^{\prime} ; \omega\right) & =\frac{1}{\mathcal{N} V_{0}} \int d \mathbf{r} d \mathbf{r}^{\prime} \mathrm{e}^{-i \mathbf{q} \cdot \mathbf{r}} \mathcal{X}_{a b}\left(\mathbf{r}, \mathbf{r}^{\prime} ; \omega\right) \mathrm{e}^{i \mathbf{q}^{\prime} \cdot \mathbf{r}^{\prime}} \\
& \equiv \frac{1}{V_{0}} S(\mathbf{q}) S^{*}\left(\mathbf{q}^{\prime}\right) \hat{\mathcal{X}}_{a b}\left(\mathbf{q}, \mathbf{q}^{\prime} ; \omega\right)
\end{aligned}
$$

where $V_{0}$ is the volume of the elementary crystal cell and

$$
S(\mathbf{q}) \equiv \int d \mathbf{r} \mathrm{e}^{-i \mathbf{q r}}|\phi(\mathbf{r})|^{2}
$$

is a form factor (which can be set equal to unity for all practical purposes). Applying a suitable unitary transformation [cf. Eq. (A23)] which renders the matrix $T\left(\Omega_{i}\right)$ of Eq. (13) diagonal, one gets for its lattice Fourier transform:

$$
\begin{aligned}
\bar{T}_{a b}(\mathbf{k}) & =\frac{1}{\mathcal{N}} \sum_{i} \mathrm{e}^{i \mathbf{k} \cdot \mathbf{R}_{i}} \bar{T}_{a b}\left(\Omega_{i}\right) \\
& =\left(\begin{array}{cccc}
\Delta(\mathbf{k}) & 0 & 0 & 0 \\
0 & \Delta(\mathbf{k}-\mathbf{Q}) & 0 & 0 \\
0 & 0 & \Delta(\mathbf{k}) & 0 \\
0 & 0 & 0 & \Delta(\mathbf{k}+\mathbf{Q})
\end{array}\right)
\end{aligned}
$$

$\Delta(\mathbf{k})$ being the lattice Kronecker delta function. In the new basis, we thus obtain for the non-interacting correlation function the expression:

$$
\begin{aligned}
\hat{\mathcal{X}}_{a b}^{(0)}\left(\mathbf{q}, \mathbf{q}^{\prime} ; \omega\right) & =\frac{1}{4 \mathcal{N}} \sum_{\mathbf{k k}^{\prime}}^{B Z} \sum_{a^{\prime}, b^{\prime}} \sum_{r, r^{\prime}} \bar{T}_{a a^{\prime}}\left(\mathbf{k}-\mathbf{k}^{\prime}-\mathbf{q}\right) \bar{T}_{b^{\prime} b}\left(\mathbf{k}^{\prime}-\mathbf{k}+\mathbf{q}^{\prime}\right) \\
& \times \bar{F}_{r^{\prime}, r}^{a^{\prime}}\left(\mathbf{k}^{\prime}, \mathbf{k}\right) \bar{F}_{r, r^{\prime}}^{b^{\prime}}\left(\mathbf{k}, \mathbf{k}^{\prime}\right) \mathcal{F}_{r, r^{\prime}}\left(\mathbf{k}, \mathbf{k}^{\prime}, \omega\right)
\end{aligned}
$$

where the overbar denotes matrices transformed according to the above unitary transformation [cf. Eqs. (A19) and (A23)]. In this way, the integral equation (11) reduces to the form:

$$
\begin{aligned}
\hat{\mathcal{X}}_{a b}\left(\mathbf{q}, \mathbf{q}^{\prime} ; \omega\right) & =\hat{\mathcal{X}}_{a b}^{(0)}\left(\mathbf{q}, \mathbf{q}^{\prime} ; \omega\right) \\
& +2 U \sum_{a^{\prime} b^{\prime}} \sum_{\mathbf{q}^{\prime \prime}} \hat{\mathcal{X}}_{a a^{\prime}}^{(0)}\left(\mathbf{q}, \mathbf{q}^{\prime \prime} ; \omega\right) \bar{\epsilon}_{a^{\prime} b^{\prime}} \hat{\mathcal{X}}_{b^{\prime} b}\left(\mathbf{q}^{\prime \prime}, \mathbf{q}^{\prime} ; \omega\right)
\end{aligned}
$$


with $\bar{\epsilon}$ given by Eq. (A22). This equation can be solved by the methods of Appendix A, yielding the closed-form expression:

$$
\begin{aligned}
\hat{\mathcal{X}}_{a b}\left(\mathbf{q}+\gamma_{a} \mathbf{Q}, \mathbf{q}^{\prime} ; \omega\right) & =\sum_{a^{\prime}}[\mathbf{1}+X(\mathbf{q}, \omega)]_{a a^{\prime}}^{-1} \\
& \times X_{a^{\prime} b}^{(0)}\left(\mathbf{q}+\gamma_{a^{\prime}} \mathbf{Q} ; \omega \mid \mathbf{Q}\right) \Delta\left(\mathbf{q}-\mathbf{q}^{\prime}-\gamma_{b} \mathbf{Q}\right)
\end{aligned}
$$

where 1 is the $4 \times 4$ unit matrix, the matrix $X(\mathbf{q}, \omega)$ is defined by Eq. (A30), and with the notation $\gamma_{a}=0$ for $a=0,2, \gamma_{a}=-1$ for $a=1$, and $\gamma_{a}=1$ for $a=3$. Although still expressed in the transformed basis, Eq. (19) is the desired expression for the Fourier transform of the generalized correlation function, which holds within the RPA approximation for any value of $\mathbf{Q}$.

The spin-wave dispersion relation can eventually be obtained by searching for the zeros of the inverse matrix on the right-hand side of Eq. (19), which is equivalent to imposing the condition:

$$
\operatorname{det}[\mathbf{1}+X(\mathbf{q}, \omega)]=0 .
$$

It can be verified that the condition (20) can be mapped onto the result reported in Refl, where the dispersion relation has then been obtained numerically for chosen values of $\mathbf{Q}$. In the present paper we proceed instead to deriving the analytic form of the dispersion relation for small values of the parameter $t / U$ of the Hubbard Hamiltonian.

To this end, it is convenient to rewrite first the matrix $X$ in Eq. $(20)$ in a more conventional basis identified by the labels $(0,+,-, z)$, with $\sigma^{ \pm}=\left(\sigma^{x} \pm i \sigma^{y}\right) / \sqrt{2}$. The matrix $\mathbf{1}+X(\mathbf{q}, \omega)$ is then transformed into:

$$
M(\mathbf{q}, \omega)=\mathbf{1}+2 U\left(\begin{array}{llll}
-\mathcal{X}_{0}^{0,0}(\mathbf{q}, \omega) & \mathcal{X}_{0}^{0,-}(\mathbf{q}, \omega) & \mathcal{X}_{0}^{0,+}(\mathbf{q}, \omega) & \mathcal{X}_{0}^{0, z}(\mathbf{q}, \omega) \\
-\mathcal{X}_{0}^{+, 0}(\mathbf{q}, \omega) & \mathcal{X}_{0}^{+,-}(\mathbf{q}, \omega) & \mathcal{X}_{0}^{+,+}(\mathbf{q}, \omega) & \mathcal{X}_{0}^{+,,}(\mathbf{q}, \omega) \\
-\mathcal{X}_{0}^{-, 0}(\mathbf{q}, \omega) & \mathcal{X}_{0}^{-,-}(\mathbf{q}, \omega) & \mathcal{X}_{0}^{-,+}(\mathbf{q}, \omega) & \mathcal{X}_{0}^{-,, z}(\mathbf{q}, \omega) \\
-\mathcal{X}_{0}^{z, 0}(\mathbf{q}, \omega) & \mathcal{X}_{0}^{z,-}(\mathbf{q}, \omega) & \mathcal{X}_{0}^{z,+}(\mathbf{q}, \omega) & \mathcal{X}_{0}^{z, z}(\mathbf{q}, \omega)
\end{array}\right)
$$

where now

$$
\mathcal{X}_{0}^{\alpha, \beta}(\mathbf{q}, \omega)=\frac{1}{4 \mathcal{N}} \sum_{r, r^{\prime}} \sum_{\mathbf{k}}^{B Z} F_{r^{\prime}, r}^{\alpha}(\mathbf{k}-\mathbf{q}, \mathbf{k}) F_{r, r^{\prime}}^{\beta}(\mathbf{k}, \mathbf{k}-\mathbf{q}) \mathcal{F}_{r, r^{\prime}}(\mathbf{k}, \mathbf{k}-\mathbf{q}, \omega)
$$

and

$$
F_{r, r^{\prime}}^{\mu}\left(\mathbf{k}, \mathbf{k}^{\prime}\right)=\sum_{\xi, \xi^{\prime}} W_{r, \xi}^{\dagger}(\mathbf{k}) \sigma_{\xi, \xi^{\prime}}^{\mu} W_{\xi^{\prime}, r^{\prime}}\left(\mathbf{k}^{\prime}\right)
$$

with $\sigma^{+}=\sqrt{2}\left(\begin{array}{ll}0 & 1 \\ 0 & 0\end{array}\right)$ and $\sigma^{-}=\sqrt{2}\left(\begin{array}{ll}0 & 0 \\ 1 & 0\end{array}\right)$. Note that two columns in the expression (21) appear interchanged with respect to the original order, owing to the presence in the final basis of the tensor

$$
\tilde{\epsilon}=\left(\begin{array}{rrrr}
1 & 0 & 0 & 0 \\
0 & 0 & -1 & 0 \\
0 & -1 & 0 & 0 \\
0 & 0 & 0 & -1
\end{array}\right)
$$

in the place of $\bar{\epsilon}$ given by Eq. (A22).

We pass now to perform the small $t / U$ expansion of the spin-wave dispersion relation obtained from the condition $\operatorname{det} M(\mathbf{q}, \omega)=0$. 


\section{EXPANSION IN THE SMALL PARAMETER $t / U$}

In this Section, we obtain explicitly the spin wave dispersion relation to second order in the small parameter $t / U$, from the general condition $\operatorname{det} M(\mathbf{q}, \omega)=0$ obtained in Section 2 within the RPA approximation for the zerotemperature broken-symmetry phase with a generic incommensurate wave vector $\mathbf{Q}$. To this end, we will preliminary expand the self-consistency parameters of the mean-field Hamiltonian, as well as the equations they satisfy, at the relevant order in $t / U$; we will then expand the matrix elements of the matrix $M(\mathbf{q}, \omega)$ defined by Eq. (21) at the relevant order in $t / U$.

\section{A. Mean-field equations}

The mean-field equations for a single-band Hubbard Hamiltonian in the presence of an incommensurate spiral spin structure have been discussed in Ref.16. Introducing a local set of spin quantization axis, with the $z$ axis transformed locally into the axis specified by the spherical angles $\Omega_{i} \equiv\left(\theta_{i}=\mathbf{Q} \cdot \mathbf{R}_{i}, \varphi_{i}=0\right)$ at site $i$, one transforms the destruction operators $c_{i \alpha}$ according to Eq. (A6) and performs the (Hartree-Fock) mean-field decoupling of the Hubbard Hamiltonian, yielding

$$
H(\mathbf{Q})=\sum_{\mathbf{k}}^{B Z} \sum_{\xi, \xi^{\prime}} d_{\mathbf{k} \xi}^{\dagger} \mathcal{H}_{\xi, \xi^{\prime}}(\mathbf{k}) d_{\mathbf{k} \xi^{\prime}}-U \mathcal{N}\left(m_{1}^{2}-m_{2}^{2}\right)
$$

with $\xi, \xi^{\prime}=(+,-)$ and where $\mathcal{H}$ is the $2 \times 2$ matrix

$$
\mathcal{H}(\mathbf{k})=\left(\begin{array}{cc}
\epsilon_{0}-\mu+t T_{e}(\mathbf{k})+U\left(m_{1}-m_{2}\right) & -i t T_{o}(\mathbf{k}) \\
i t T_{o}(\mathbf{k}) & \epsilon_{0}-\mu+t T_{e}(\mathbf{k})+U\left(m_{1}+m_{2}\right)
\end{array}\right) .
$$

In the above expressions, $\epsilon_{0}$ is the site energy, $\mu$ the chemical potential, $m_{1}$ and $m_{2}$ represent the occupation number and magnetization along the local quantization axis, respectively, and $T_{e / o}(\mathbf{k}) \operatorname{read} 16$

$$
\begin{aligned}
& T_{e}(\mathbf{k})=2\left[\cos k_{x} \cos \left(\frac{Q_{x}}{2}\right)+\cos k_{y} \cos \left(\frac{Q_{y}}{2}\right)\right] \\
& T_{o}(\mathbf{k})=2\left[\sin k_{x} \sin \left(\frac{Q_{x}}{2}\right)+\sin k_{y} \sin \left(\frac{Q_{y}}{2}\right)\right] .
\end{aligned}
$$

The eigenvalues and eigenvectors of the matrix (26) are thus given by:

$$
\epsilon_{r}(\mathbf{k})=\epsilon_{0}-\mu+t T_{e}(\mathbf{k})+U m_{1}+(-1)^{r} \sqrt{U^{2} m_{2}^{2}+t^{2} T_{o}(\mathbf{k})^{2}}
$$

$(r=1,2)$ and

$$
\begin{aligned}
& W_{1}(\mathbf{k})=\frac{1}{N_{1}(\mathbf{k})}\left(-i \frac{\frac{t}{U} \frac{1}{m_{2}} T_{o}(\mathbf{k})}{1+\sqrt{1+\left(\frac{t}{U}\right)^{2} \frac{1}{m_{2}^{2}} T_{o}(\mathbf{k})^{2}}}\right) \\
& W_{2}(\mathbf{k})=\frac{1}{N_{2}(\mathbf{k})}\left(\begin{array}{c}
-i \frac{\frac{t}{U} \frac{1}{m_{2}} T_{o}(\mathbf{k})}{1+\sqrt{1+\left(\frac{t}{U}\right)^{2} \frac{1}{m_{2}^{2}} T_{o}(\mathbf{k})^{2}}} \\
1
\end{array}\right)
\end{aligned}
$$

where $N_{r}(\mathbf{k})$ stands for the normalization factor.

The parameters $m_{1}, m_{2}$, and $\mathbf{Q}$ of the mean-field Hamiltonian (25) are obtained, as usual, by minimizing the average value of the Hamiltonian with respect to the parameters themselves. One obtains: 


$$
m_{1}=\frac{1}{2 \mathcal{N}} \sum_{\mathbf{k}}^{B Z} \sum_{r} f_{F}\left(\epsilon_{r}(\mathbf{k})\right)=\frac{1+\delta}{2},
$$

where $f_{F}(\epsilon)$ is the (zero-temperature) Fermi function and $\delta$ is the doping parameter,

$$
m_{2}=\frac{1}{2 \mathcal{N}} \sum_{\mathbf{k}}^{B Z} \sum_{\xi} \sum_{r} \xi W_{r, \xi}^{\dagger}(\mathbf{k}) W_{\xi, r}(\mathbf{k}) f_{F}\left(\epsilon_{r}(\mathbf{k})\right),
$$

and

$$
\sum_{\mathbf{k}}^{B Z} \sum_{\xi, \xi^{\prime}} \vec{\nabla}_{\mathbf{Q}} \mathcal{H}_{\xi, \xi^{\prime}}(\mathbf{k}) \sum_{r} W_{r, \xi}^{\dagger}(\mathbf{k}) W_{\xi^{\prime}, r}(\mathbf{k}) f_{F}\left(\epsilon_{r}(\mathbf{k})\right)=0 .
$$

In the following, we shall restrict to the diagonal solution $\mathbf{Q}=Q(1,1)$, since it is known to be favored for sufficiently small values of $t / U 11$ Accordingly, at the order we are considering of the small parameter $t / U$ we expand formally:

$$
\begin{aligned}
\epsilon_{r}(\mathbf{k}) & =U\left[\epsilon_{r}^{(0)}(\mathbf{k})-\mu^{(0)}+\left(\frac{t}{U}\right)\left(\epsilon_{r}^{(1)}(\mathbf{k})-\mu^{(1)}\right)\right. \\
& \left.+\left(\frac{t}{U}\right)^{2}\left(\epsilon_{r}^{(2)}(\mathbf{k})-\mu^{(2)}\right)+\cdots\right]
\end{aligned}
$$

as well as

$$
m_{2}=m_{2}^{(0)}+\left(\frac{t}{U}\right) m_{2}^{(1)}+\left(\frac{t}{U}\right)^{2} m_{2}^{(2)}+\cdots
$$

while, at the relevant order we can take

$$
\begin{aligned}
& W_{1}(\mathbf{k})=\frac{1}{N_{r}(\mathbf{k})}\left(\begin{array}{c}
1 \\
-i \frac{t}{U} \frac{T_{o}(\mathbf{k})}{2 m_{2}^{(0)}}
\end{array}\right) \\
& W_{2}(\mathbf{k})=\frac{1}{N_{r}(\mathbf{k})}\left(\begin{array}{c}
-i \frac{t}{U} \frac{T_{o}(\mathbf{k})}{2 m_{2}^{(0)}} \\
1
\end{array}\right)
\end{aligned}
$$

where

$$
\frac{1}{N_{r}(\mathbf{k})^{2}}=1-\left(\frac{t}{U}\right)^{2}\left(\frac{T_{o}(\mathbf{k})}{2 m_{2}^{(0)}}\right)^{2}+\mathcal{O}\left(\left(\frac{t}{U}\right)^{3}\right)
$$

is independent from $r$. Note that it is sufficient to retain the lowest-order term $m_{2}^{(0)}$ in the above equations. The parameter $m_{1}$, on the other hand, is given by Eq. (32) and is thus formally independent from $t / U$ (we anticipate, however, that the doping parameter $\delta$ will turn out to be at most of the order $t / U$ for our expansions to be internally consistent).

The coefficients $\epsilon_{r}^{(n)}(\mathbf{k})$ of Eq. (35) with $n=0,1,2, \cdots$ can be readily obtained from the expression (29) (where we may set $\epsilon_{0}=0$ for simplicity) in terms of the $m_{2}^{(n)}$ (for given $\mathbf{Q}$ ). The value of $\mu^{(0)}$ can also be readily obtained in terms of $\epsilon_{r}^{(0)}$ (cf. Appendix B). The remaining coefficients of the expansions (35) and (36) can further be determined by solving the coupled equations for the self-consistency parameters according to the methods developed in the Appendices B and C. In particular, for $\delta>0$ we obtain:

$$
\begin{aligned}
& m_{2}^{(0)}=\frac{1}{2}(1-\delta) \\
& m_{2}^{(1)}=0 \\
& m_{2}^{(2)}=-4 \sin ^{2}(Q / 2)+\mathcal{O}(\delta),
\end{aligned}
$$




$$
\begin{aligned}
& \mu^{(0)}-m_{2}^{(0)}=\frac{1}{2}(1+\delta) \\
& \mu^{(1)}-m_{2}^{(1)}=\cos (Q / 2)\left[4-4 \pi \delta+\mathcal{O}\left(\delta^{2}\right)\right] \\
& \mu^{(2)}-m_{2}^{(2)}=0+\mathcal{O}(\delta)
\end{aligned}
$$

and

$$
\begin{aligned}
& \epsilon_{r}^{(0)}(\mathbf{k})=\frac{1}{2}\left[(1+\delta)+(-1)^{r}(1-\delta)\right] \\
& \epsilon_{r}^{(1)}(\mathbf{k})=T_{e}(\mathbf{k}) \\
& \epsilon_{r}^{(2)}(\mathbf{k})=(-1)^{r}\left[T_{o}^{2}(\mathbf{k})-4 \sin ^{2}(Q / 2)\right]+\mathcal{O}(\delta),
\end{aligned}
$$

where for the spiral configuration we are considering $Q$ is determined by the condition

$$
\cos (Q / 2)=\frac{-U \delta}{2 t}+\mathcal{O}(t / U)
$$

with $\delta \leq 2(t / U)+\mathcal{O}\left((t / U)^{2}\right)$, as anticipated. (The other allowed solution $\sin (Q / 2)=0$ describes instead the ferromagnetic case.) As discussed in Appendix B, the above results have been obtained with the further assumption that $\delta$ is small enough but not infinitesimal, i.e., $\delta$ satisfies the condition $(t / U)^{2}<<\delta$. The case $\delta=0$, on the other hand, can be considered separately. Note that Eq. (43) implies that in the spiral phase $\delta$ is at most of the order $t / U$. This property has to be taken into account to get a consistent expansion up to the desired order in $t / U$.

\section{B. Susceptibilities and spin-wave dispersion}

Before performing the $t / U$ expansion of the matrix elements of the non-interacting susceptibility tensor (22) to get the spin-wave dispersion relation, it is convenient to exploit some symmetry properties that reduce the number of matrix elements to be considered. Specifically, from the property

$$
\mathcal{F}_{r, r^{\prime}}(\mathbf{k}, \mathbf{k}-\mathbf{q}, \omega)=\mathcal{F}_{r^{\prime}, r}(\mathbf{k}-\mathbf{q}, \mathbf{k},-\omega)
$$

it follows that:

$$
\mathcal{X}_{0}^{\alpha, \beta}(\mathbf{q}, \omega)=\mathcal{X}_{0}^{\beta, \alpha}(-\mathbf{q},-\omega) .
$$

By direct inspection it can also be verified that:

$$
\begin{aligned}
& \mathcal{X}_{0}^{0,-}(\mathbf{q}, \omega)=-\mathcal{X}_{0}^{+, 0}(\mathbf{q}, \omega) \\
& \mathcal{X}_{0}^{z,-}(\mathbf{q}, \omega)=-\mathcal{X}_{0}^{+,, z}(\mathbf{q}, \omega) \\
& \mathcal{X}_{0}^{+,+}(\mathbf{q}, \omega)=\mathcal{X}_{0}^{-,-}(\mathbf{q}, \omega)
\end{aligned}
$$

In this way, the matrix (21) acquires the simplified form:

$$
M(\mathbf{q}, \omega)=\left(\begin{array}{cccc}
1-a(\mathbf{q}, \omega) & i b(\mathbf{q}, \omega) & -i b(-\mathbf{q},-\omega) & c(\mathbf{q}, \omega) \\
i b(\mathbf{q}, \omega) & 1+d(\mathbf{q}, \omega) & e(\mathbf{q}, \omega) & i f(\mathbf{q}, \omega) \\
-i b(-\mathbf{q},-\omega) & e(\mathbf{q}, \omega) & 1+d(-\mathbf{q},-\omega) & -i f(-\mathbf{q},-\omega) \\
-c(\mathbf{q}, \omega) & -i f(\mathbf{q}, \omega) & i f(-\mathbf{q},-\omega) & 1+g(\mathbf{q}, \omega)
\end{array}\right)
$$

where we have set

$$
\begin{aligned}
& a(\mathbf{q}, \omega)=2 U \mathcal{X}_{0}^{0,0}(\mathbf{q}, \omega) \\
& b(\mathbf{q}, \omega)=-2 i U \mathcal{X}_{0}^{0,-}(\mathbf{q}, \omega) \\
& c(\mathbf{q}, \omega)=2 U \mathcal{X}_{0}^{0, z}(\mathbf{q}, \omega) \\
& d(\mathbf{q}, \omega)=2 U \mathcal{X}_{0}^{+,-}(\mathbf{q}, \omega) \\
& e(\mathbf{q}, \omega)=2 U \mathcal{X}_{0}^{+,+}(\mathbf{q}, \omega) \\
& f(\mathbf{q}, \omega)=-2 i U \mathcal{X}_{0}^{+, z}(\mathbf{q}, \omega) \\
& g(\mathbf{q}, \omega)=2 U \mathcal{X}_{0}^{z, z}(\mathbf{q}, \omega) .
\end{aligned}
$$


Entering then the expansions (37)-(39) for the eigenvectors of the mean-field Hamiltonian (with $m_{2}^{(0)}$ given by Eq. (40)) into the definition (23), we obtain the expressions for the relevant matrix elements of the non-interacting susceptibility tensor (22) reported in Appendix B at the order in $t / U$ we are considering. Utilizing further the method developed in Appendix $\mathrm{C}$ to perform the $\mathbf{k}$ summation when the doping parameter $\delta$ is small, we obtain eventually the following expressions for the matrix elements (48):

$$
\begin{aligned}
& a(\mathbf{q}, \omega)=a(\mathbf{q})=\frac{-1}{2 \cos (Q / 2)\left(\cos q_{x}+\cos q_{y}-2\right)} \frac{\delta U}{t}+\mathcal{O}(t / U), \\
& b(\mathbf{q}, \omega)=b(\mathbf{q})=\frac{-\sin (Q / 2)\left(\sin q_{x}+\sin q_{y}\right)}{\sqrt{2} \cos (Q / 2)\left(\cos q_{x}+\cos q_{y}-2\right)} \delta+\mathcal{O}\left((t / U)^{2}\right), \\
& c(\mathbf{q}, \omega)=c(\mathbf{q})=-a(\mathbf{q}, \omega)+\mathcal{O}(t / U) \\
& e(\mathbf{q}, \omega)=e(\mathbf{q})=-4\left(\frac{t}{U}\right)^{2} \sin ^{2}(Q / 2)\left(\cos q_{x}+\cos q_{y}\right)+\mathcal{O}\left((t / U)^{3}\right), \\
& f(\mathbf{q}, \omega)=f(\mathbf{q})=b(\mathbf{q}, \omega)+\mathcal{O}\left((t / U)^{2}\right), \\
& g(\mathbf{q}, \omega)=g(\mathbf{q})=a(\mathbf{q}, \omega)+\mathcal{O}(t / U),
\end{aligned}
$$

as well as

$$
\begin{aligned}
d(\mathbf{q}, \omega) & \equiv-1-\tilde{\omega}+\alpha(\mathbf{q})+\mathcal{O}\left((t / U)^{3}\right) \\
& =-1-\tilde{\omega}+\left(\frac{t}{U}\right)^{2} 4 \cos ^{2}(Q / 2)\left(\cos q_{x}+\cos q_{y}-2\right)+8\left(\frac{t}{U}\right)^{2} \sin ^{2}(Q / 2) \\
& +2\left(\frac{t}{U}\right) \delta \cos (Q / 2)\left(\cos q_{x}+\cos q_{y}-2\right) \\
& -2\left(\frac{t}{U}\right) \delta \frac{\sin ^{2}(Q / 2)\left(\sin q_{x}+\sin q_{y}\right)^{2}}{\cos (Q / 2)\left(\cos q_{x}+\cos q_{y}-2\right)}+\mathcal{O}\left((t / U)^{3}\right)
\end{aligned}
$$

where $\tilde{\omega} \equiv \omega / U$ will turn out to be of order $t^{2} / U^{2}$ at the spin-wave poles.

To obtain the above expressions, we have considered only the real part of the functions (A16). This is definitely possible for every pairs of bands when we restrict to values of $\mathbf{q}$ such that $|\mathbf{q}| \gg k_{F}$, where $k_{F}$ (by our definition) is the maximum value of the function $k(\phi)$ introduced in Appendix $\mathrm{C}$, which coincides with the Fermi momentum for small $\delta$. Since we have shown in the same Appendix that $k(\phi) \sim \sqrt{\delta} \sim \sqrt{t / U}$, taking the $\mathbf{q} \rightarrow 0$ limit implies letting $t / U$ to vanish before $\mathbf{q}$.

Note also that, although the expressions (49) and (50) have been calculated at different orders in $t / U$, the ensuing expression for the determinant of the matrix (47) is obtained consistently at the fourth order in $t / U$, as required for the frequency of the spin-wave mode to be of the order of $t^{2} / U$. In fact, by writing the determinant explicitly we obtain:

$$
\begin{aligned}
& {\left[(1-a(\mathbf{q}))(1+a(\mathbf{q}))+a^{2}(\mathbf{q})+\mathcal{O}(t / U)\right]} \\
& \times\left[(-\tilde{\omega}+\alpha(\mathbf{q}))(\tilde{\omega}+\alpha(\mathbf{q}))-e^{2}(\mathbf{q})+\mathcal{O}\left((t / U)^{5}\right)\right] \\
& +2[1-a(\mathbf{q})+\mathcal{O}(t / U)]\left[b^{2}(\mathbf{q})+\mathcal{O}\left((t / U)^{3}\right)\right]\left[e(\mathbf{q})-\alpha(\mathbf{q})+\mathcal{O}\left((t / U)^{3}\right)\right] \\
& -2[1+a(\mathbf{q})+\mathcal{O}(t / U)]\left[b^{2}(\mathbf{q})+\mathcal{O}\left((t / U)^{3}\right)\right]\left[e(\mathbf{q})-\alpha(\mathbf{q})+\mathcal{O}\left((t / U)^{3}\right)\right] \\
& +4[a(\mathbf{q})+\mathcal{O}(t / U)]\left[b^{2}(\mathbf{q})+\mathcal{O}\left((t / U)^{3}\right)\right]\left[e(\mathbf{q})-\alpha(\mathbf{q})+\mathcal{O}\left((t / U)^{3}\right)\right]=0 .
\end{aligned}
$$

Note that the last three terms on the left-hand side add up to zero at the fourth order in $t / U$ we are considering. We are thus left with the expression

$$
\tilde{\omega}^{2}-\alpha^{2}(\mathbf{q})+e^{2}(\mathbf{q})+\mathcal{O}\left((t / U)^{5}\right)=0
$$

yielding 


$$
\omega^{2}(\mathbf{q})=U^{2}\left(\alpha^{2}(\mathbf{q})-e^{2}(\mathbf{q})\right)+\mathcal{O}\left(t^{5} / U^{3}\right)
$$

Taking into account the expressions for $\alpha(\mathbf{q})$ and $e(\mathbf{q})$ given above, we get eventually the desired spin-wave dispersion relation, in the form:

$$
\begin{aligned}
\omega^{2}(\mathbf{q}) & =16 \frac{t^{4}}{U^{2}} \cos Q\left(\cos q_{x}+\cos q_{y}-2\right)\left[\cos q_{x}+\cos q_{y}-2 \cos Q\right] \\
& +16 \frac{t^{3}}{U} \delta\left[\cos (Q / 2)\left(\cos q_{x}+\cos q_{y}-2\right)-\frac{\sin ^{2}(Q / 2)\left(\sin q_{x}+\sin q_{y}\right)^{2}}{\cos (Q / 2)\left(\cos q_{x}+\cos q_{y}-2\right)}\right] \\
& \times\left[\cos ^{2}(Q / 2)\left(\cos q_{x}+\cos q_{y}-2\right)+2 \sin ^{2}(Q / 2)\right] \\
& +4 \delta^{2} t^{2}\left[\cos (Q / 2)\left(\cos q_{x}+\cos q_{y}-2\right)-\frac{\sin ^{2}(Q / 2)\left(\sin q_{x}+\sin q_{y}\right)^{2}}{\cos (Q / 2)\left(\cos q_{x}+\cos q_{y}-2\right)}\right]^{2} .
\end{aligned}
$$

To obtain the physical dispersion relation, however, there remains to enter in the above expression the relation among $Q, \delta$, and $t / U$ as given by the mean-field condition (43), as discussed in the next Section.

\section{RESULTS AND DISCUSSION}

In this Section, we discuss the physical consequences of the dispersion relation (54) for spin-wave excitations over an incommensurate (diagonal) spiral magnetic configuration of a two-dimensional Hubbard Hamiltonian.

We have already remarked that the dispersion relation (54) is not yet in its final form, since the connection among $Q, \delta$, and $t / U$ needs still to be specified. Before considering the general case, however, it is interesting to recover from Eq. (54) the spin-wave dispersion relations corresponding to the limiting cases of an antiferromagnet and of a ferromagnet.

When $\delta=0$, Eq. (B14) yields $\cos (Q / 2)=0$, that is $\mathbf{Q}=\mathbf{Q}_{A F}=(\pi, \pi)$. In this case we obtain from Eq. (54) (by setting $\delta=0$ identically therein):

$$
\omega(\mathbf{q})=J_{\mathrm{eff}}^{(A F)}\left[4-\left(\cos q_{x}+\cos q_{y}\right)^{2}\right]^{\frac{1}{2}}
$$

with $J_{\mathrm{eff}}^{(A F)}=4 t^{2} / U$. This result coincides with the spin-wave dispersion relation of a two-dimensional Heisenberg antiferromagnet at leading order in $t / U .17$

When $\mathbf{Q}=(2 \pi, 2 \pi)$ and $\delta$ arbitrary, we obtain instead from Eq. (54)

$$
\omega(\mathbf{q})=\frac{4 t^{2}}{U}\left|\left(1-\frac{\delta U}{2 t}\right)\right|\left(2-\cos q_{x}-\cos q_{y}\right),
$$

which coincides with the spin-wave dispersion relation of a nearest-neighbor Heisenberg ferromagnet with $J_{\text {eff }}^{(F)}=$ $4 t^{2} / U[1-\delta U /(2 t)]$. Note that $J_{\text {eff }}^{(F)}$ is negative since the (mean-field) ferromagnetic solution is actually stable when $\delta \geq 2 t / U$.

In the general case of a (diagonal) spiral spin configuration, the relation among $Q, \delta$, and $t / U$ is given by Eq. (B14), that is, $\delta=-(2 t / U) \cos Q / 2+\mathcal{O}\left((t / U)^{2}\right)$ at the leading order in $t / U$. Eliminating $Q$ in favor of $\delta$ and $t / U$ via this relation in Eq. (54), we obtain eventually the following expression:

$$
\omega(\mathbf{q})=J_{\mathrm{eff}}\left\{\left[2-\frac{\left(\sin q_{x}+\sin q_{y}\right)^{2}}{2-\cos q_{x}-\cos q_{y}}\right]^{2}-\left(\cos q_{x}+\cos q_{y}\right)^{2}\right\}^{\frac{1}{2}}
$$

where now the effective exchange integral is given by 


$$
J_{\text {eff }}=\frac{4 t^{2}}{U} \sin ^{2}(Q / 2)=\frac{4 t^{2}}{U}\left(1-\left(\frac{U}{2 t} \delta\right)^{2}\right)=\frac{4 t^{2}}{U}\left(1-\left(\frac{\delta}{\delta_{c}}\right)^{2}\right)
$$

with $\delta_{c} \equiv 2 t / U$. Equation (57) constitutes the main result of this paper. Note that when $\delta$ reaches the critical value $\delta_{c}, J_{\text {eff }}$ vanishes. Past this value, the spiral solution evolves into the ferromagnetic solution, which becomes the stable solution (at the mean-field level).

The real and imaginary parts of $\omega(\mathbf{q})$ (in units of $J_{\text {eff }}$ ), as obtained from the analytic expression (57), are plotted in Figs. 1(a) and 1(b), respectively, over the two-dimensional Brillouin zone.
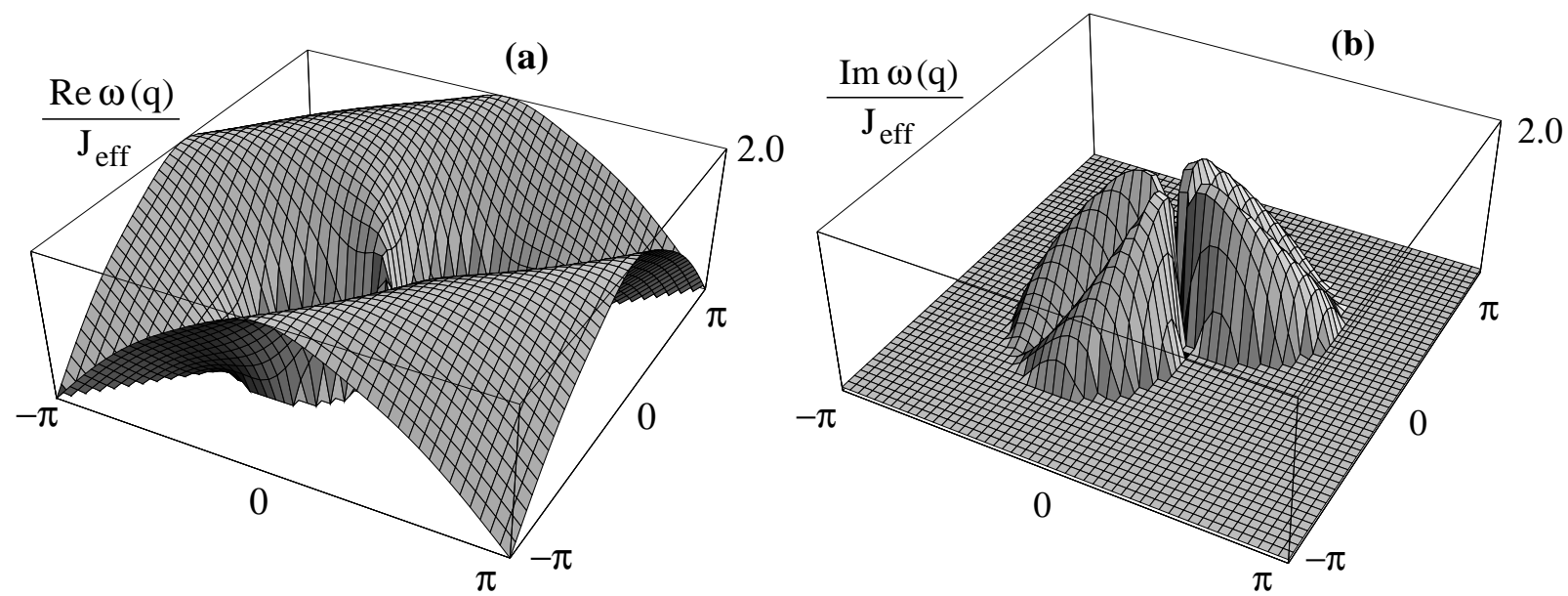

FIG. 1. Real (a) and imaginary (b) part of the spin-wave dispersion relation (57) (in units of $J_{\text {eff }}$ ), obtained with a diagonal-spiral spin configuration background. Note that the $Q$-dependence is contained in $J_{\text {eff }}$ only.

It is clear from the expression (57) that $\omega(\mathbf{q})$ is either real or purely imaginary, so that, when its real part is nonvanishing, its imaginary part vanishes identically, and viceversa. Actually, this is true in the region of the Brillouin zone where our expansion holds, namely, for $|\mathbf{q}| \gg k_{F}$ [cf. the discussion below Eq. (5月]. For $|\mathbf{q}|<k_{F}$, the spin-wave dispersion acquires a damping due to the mixing with the particle-hole continuum. 18 An exception is represented by the line $q_{x}=q_{y}$, along which the real and imaginary parts vanish simultaneously. The softening of the dispersion relation along the whole line $q_{x}=q_{y}$ and not only when $\mathbf{q}=0$ and $\mathbf{q}=\mathbf{Q}$ (as one would expect on general grounds in the presence of a spiral spin configurationt) corresponds to the fact that $\omega(\mathbf{q})$ given by Eq. (57) can be cast in the form $\omega(\mathbf{q})=f(\mathbf{Q}) g_{\hat{Q}}(\mathbf{q})$; as $\omega(\mathbf{q}=\mathbf{Q})=0$ invariably, it follows that $g_{\hat{Q}}(\mathbf{q}=\mathbf{Q})=0$ since $g_{\hat{Q}}(\mathbf{q})$ does not depend on $|\mathbf{Q}|$. This implies that $g_{\hat{Q}}\left(q_{x}=q_{y}\right) \equiv 0$ identically.

One expects this result to be modified, however, at higher order in $t / U$. In this respect, it is interesting to compare with the results obtained by Brenig by solving numerically the condition (20) directly, without performing the expansion in the small parameter $t / U$. One sees, in particular, from Fig. 6 of Brenig's paper (obtained for $\delta=0.075$ ) that $\omega(\mathbf{q})$ remains indeed finite along $q_{x}=q_{y}$ already when $t / U=0.1$.

Figure 2 shows the region of the Brillouin zone (shaded area) where $\omega(\mathbf{q})$ is overdamped (purely imaginary) (with the region about $\mathbf{q}=0$ excluded according to the argument given in Section 3). A finite region of the Brillouin zone where the dispersion relation is overdamped is also reported in Ref.U, even though a direct comparison with our results is not possible owing to the different ranges of the parameter $t / U$ explored. This overdamping signals an instability of the system (due to the merging into the particle-hole continuum) toward a different ground state, reflecting possibly a more complicated long-range spin (and charge) structure than the spin-spiral one considered in the present paper. Nonetheless, we expect on physical grounds that close to the boundary of the Brillouin zone (where overdamping of spin waves does not occur in our calculation) the spin-wave spectrum with small wavelength obtained by our approach would survive the inclusion of more complicated long-range spin structures.

It is also interesting to compare the form of the dispersion relation (57) with the spin-wave dispersion relation obtained with the Heisenberg model including second and third nearest-neighbor couplings, for the same value of the incommensurability wave vector $Q$ (cf. Appendix D). This comparison is shown in Fig. 3 along the symmetry lines of the Brillouin zone and evidences marked differences between the two dispersion relations.

Returning to the dispersion relation $(57)$ for $\delta \neq 0$, we emphasize that its functional form could not be obtained from the dispersion relation (55) valid when $\delta=0$, by simply modifying the numerical values of the exchange integral 


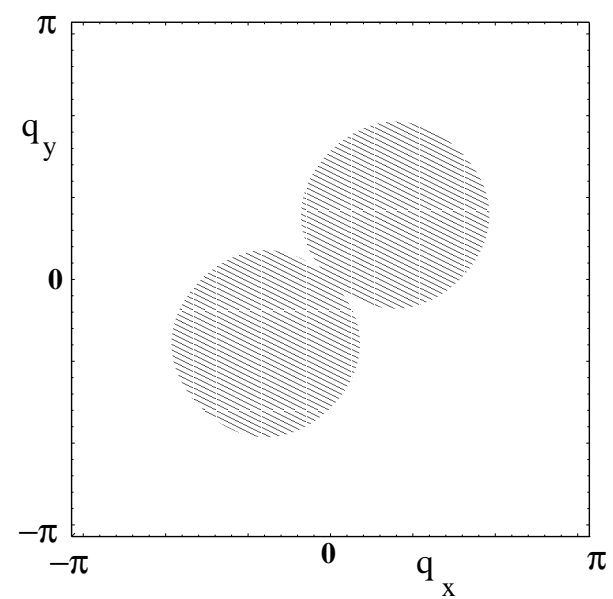

FIG. 2. Region of the two-dimensional Brillouin zone where the spin-wave dispersion is overdamped (shaded area). Note that this region does not depend on $Q$, and that the region about the center of the Brillouin zone has been excluded by construction.

therein. Nor, it would be sufficient to include a finite number of exchange integrals in the Heisenberg model to account for the finite doping, owing to the presence of an RKKY-type term in Eq. (57) which contains trigonometric functions in the denominator rather than in the numerator only (cf. Appendix E). Recall, in fact, that for practical purposes the Heisenberg model can be regarded as a fitting model which could, in principle, reproduce any type of spin-mave dispersion relation, provided a sufficiently large number of terms associated with distant neighbors were included.19 In addition, it appears fair to say that it would have been certainly difficult to guess a priori the functional form of the dispersion relation (57), by fitting the numerical results for the itinerant model onto the Heisenberg model extended to a large number of neighbors.

It is also interesting to comment on the effective exchange integral (58) retaining the $t^{2} / U$ dependence of the nearestneighbor Heisenberg model, even when couplings between far apart neighbors are considered. From a perturbative point of view, when $U>>t$ the magnetic interaction between a given lattice site and far apart neighbors is provided by the mobility of the holes in the doped configurations. In this respect, one should consider all possible configurations with empty sites distributed at random over the lattice sites, the mobility of the holes then resulting by diagonalizing the Hamiltonian in this basis. As a consequence, the magnetic exchange coupling turns out to be proportional to $t^{2} / U$ at leading order, even for coupling between sites at arbitrary distances.

As anticipated in the Introduction, the form (57) is somewhat hybrid between the one obtained with a nearestneighbor Heisenberg model (cf. Appendix D) and the long-range RKKY interaction mediated by the conduction electrons (cf. Appendix E). These two contributions to Eq. (57) cannot be separated in a clear and unambiguous way. However, it is possible to trace their origin by considering the expressions (B15) and (B16) for the relevant matrix elements of the susceptibility.

If one could set "by hand" $\mathcal{F}_{22}=0$ in those expressions, thus keeping only the interband terms, one would in fact obtain for the dispersion relation:

$$
\omega(\mathbf{q})=J_{\text {eff }}\left[4-\left(\cos q_{x}+\cos q_{y}\right)^{2}\right]^{\frac{1}{2}},
$$

which corresponds to a nearest-neighbor Heisenberg antiferromagnet with exchange coupling given by Eq. (58). Similarly, if one could set $\mathcal{F}_{21}=\mathcal{F}_{12}=0$ "by hand", thus keeping only the intraband terms, one would instead obtain an expression of the RKKY-type:

$$
\omega(\mathbf{q})=J_{\mathrm{eff}} \frac{\left(\sin q_{x}+\sin q_{y}\right)^{2}}{2-\cos q_{x}-\cos q_{y}}
$$

with $J_{\text {eff }}$ still given by Eq. (58). Note that in both cases the spectrum would be real and no overdamping would occur. Our general result (57) can then be cast in the following appealing form:

$$
\omega(\mathbf{q})=\left\{\left[J(\mathbf{q}=0)-\omega(\mathbf{q})_{R K K Y}\right]^{2}-J(\mathbf{q})^{2}\right\}^{1 / 2}
$$




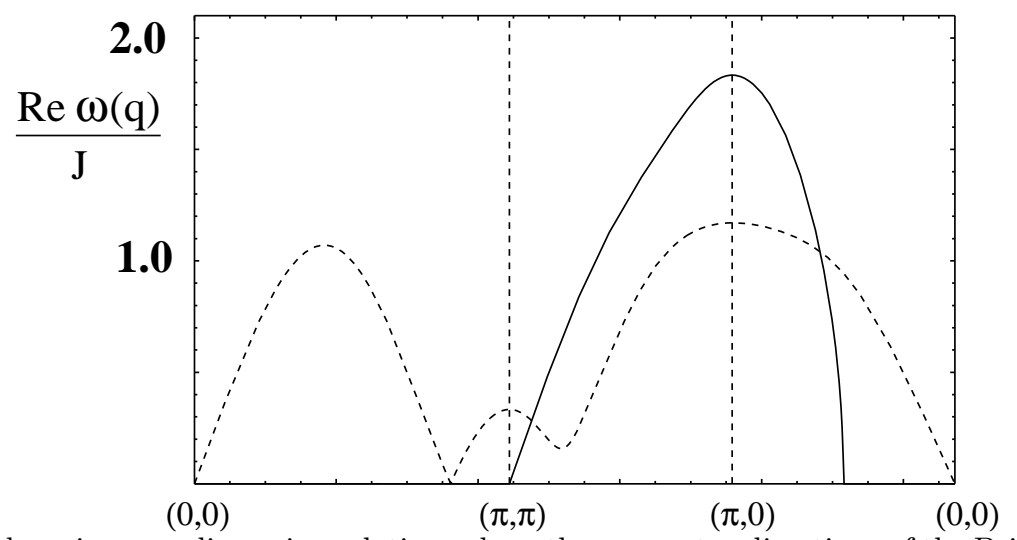

FIG. 3. Comparison of the spin-wave dispersion relations along the symmetry directions of the Brillouin zone, as obtained for the itinerant model (full line) and for the Heisenberg model with second $\left(J_{2} / J_{1}=0.2\right)$ and third $\left(J_{3} / J_{1}=0.2\right)$ nearest-neighbor couplings (broken line). Both dispersions correspond to the same value of $Q=2.556$ and are plotted in units of $J=4 t^{2} / U$ and $J_{1}$, respectively.

where $J(\mathbf{q})=J_{\text {eff }}\left(\cos q_{x}+\cos q_{y}\right)$ and $\omega(\mathbf{q})_{R K K Y}$ is given by the expression (60). Note, in particular, that if one could set $\omega(\mathbf{q})_{R K K Y}=0$, the Heisenberg form (59) would result from Eq. (61).

A final comment on the possible comparison between the spin-wave spectrum we have obtained and the available experimental data is in order. That the classical spin-wave theory (possibly with quantum corrections20) can accurately describe the spin-wave spectrum over the whole Brillouin zone for the parent compounds of high-temperature superconductors (zero doping) is well established at this point. That when carriers are added (finite doping) incommensurate spin fluctuations occur with rather short coherence length has also been well established in several materials. It is interesting to point out, in addition, that the observation of well-defined spin waves only close to the boundary of the Brillouin zone has been reported, 3 consistently with what we have obtained by our calculation. However, detailed comparison with our form (57) of the spin-wave dispersion relation with the experimental data may not be possible, because our result is valid in the asymptotic limit $U>>W$, where $W \sim 8 t$ is the bandwidth, which may not be realized for real materials.

\section{CONCLUDING REMARKS}

In this paper, we have studied the spin-wave spectrum for a two-dimensional Hubbard Hamiltonian in the presence of an incommensurate spin-spiral phase within the electronic RPA approximation in the broken-symmetry phase. We have, in particular, obtained the analytic form of this spectrum at the leading order in the small parameter $t / U$ of the Hubbard Hamiltonian. Specifically, it has been possible to obtain the spectrum in a closed form even in the presence of an incommensurate structure, owing to the peculiar symmetry which is intrinsic to the spiral phase. In this respect, starting from an alternative mean-field configuration different from the spiral one (such as, for instance, a "stripe" structure) would have not enabled us to solve for the spin wave spectrum in a closed form. By our approach, we have thus pushed the analytic results for the spin wave spectrum of an incommensurate structure as far as possible (apart, obviously, from including higher orders in the $t / U$ expansion which could still be done by our approach).

Alternatively, the spin-wave spectrum could have been obtained numerically for any value of $t / U$ without performing the small $t / U$ expansion, as already reported in Ref.U. In this way, however, it would have been rather difficult (if not impossible) to arrive at the functional form (57) for the spin-wave dispersion relation in the strong-coupling limit, which has an hybrid form between the dispersion relation for a nearest-neighbor Heisenberg model and that obtained within the (long-range) RKKY interaction in the presence of a finite doping. Owing to the itinerant character of the system, it is, in fact, the presence of a band of metallic character (crossed by the Fermi level) which generates the RKKY magnetic interaction between the localized spins associated with the (filled) lower band. This novel feature 
constitutes the main result of the present paper. We have concluded accordingly that, even for small doping, the itinerant model we have considered results in a dispersion relation $\omega(\mathbf{q})$ that cannot be effectively represented by the Heisenberg model, for which the couplings extend to a few neighbors only. This occurs because the free carriers, associated with the itinerant character of our starting Hamiltonian, necessarily introduce long-range RKKY-type magnetic interactions among the localized spins.

A serious concern, which is related to the spin-wave spectrum we have obtained, regards the instability occurring about the center of the Brillouin zone, where the spin-wave spectrum becomes purely imaginary. To overcome this point, one should possibly start from a more complicated incommensurate mean-field solution other than the spiral configuration, with a lower ground-state energy. In this way, however, one would unavoidably not obtain a closed-

form equation for the spin-wave spectrum, since the incommensurability could in general prevent it. Nonetheless, we expect on physical grounds the spin-wave spectrum we have obtained with the spiral pattern to survive inclusion of more realistic spin structures, if one considers only the region close to the boundary of the Brillouin zone, for which knowledge of the detailed form of the underlying (long-range) spin pattern appears to be less crucial.

\section{ACKNOWLEDGMENTS}

We are indebted to P. Salvi for help during the initial stage of this work, especially in developing part of the material contained in Appendix C. We are also indebted to N. Majlis, W. Brenig, and G. Aeppli for discussions.

\section{APPENDIX A: SOLUTION OF THE RPA EQUATIONS FOR THE DYNAMICAL SUSCEPTIBILITIES WITH AN INCOMMENSURATE SPIN-SPIRAL GROUND STATE}

In this Appendix, we provide the details of the derivation of the explicit form of the generalized correlation function within RPA and of the associated spin-wave dispersion relation, which were reported only schematically in Section 2.

We begin by showing how Eq. (10) of the text can be reduced to a closed-form equation for the correlation function $\mathcal{X}$ itself. To this end, we note from Eq. (5) that the following relations hold for $\mu=0$ and $\mu=z$, in the order:

$$
\begin{aligned}
\mathcal{X}_{0, \nu}\left(x, x^{\prime}\right) & =-\frac{i}{4} \sum_{\alpha^{\prime}, \beta^{\prime}} \sigma_{\alpha^{\prime}, \beta^{\prime}}^{\nu} L\left(x+, x^{\prime} \beta^{\prime} ; x^{+}+, x^{\prime+} \alpha^{\prime}\right) \\
& -\frac{i}{4} \sum_{\alpha^{\prime}, \beta^{\prime}} \sigma_{\alpha^{\prime}, \beta^{\prime}}^{\nu} L\left(x-, x^{\prime} \beta^{\prime} ; x^{+}-, x^{\prime+} \alpha^{\prime}\right)
\end{aligned}
$$

and

$$
\begin{aligned}
\mathcal{X}_{z, \nu}\left(x, x^{\prime}\right) & =-\frac{i}{4} \sum_{\alpha^{\prime}, \beta^{\prime}} \sigma_{\alpha^{\prime}, \beta^{\prime}}^{\nu} L\left(x+, x^{\prime} \beta^{\prime} ; x^{+}+, x^{\prime+} \alpha^{\prime}\right) \\
& +\frac{i}{4} \sum_{\alpha^{\prime}, \beta^{\prime}} \sigma_{\alpha^{\prime}, \beta^{\prime}}^{\nu} L\left(x-, x^{\prime} \beta^{\prime} ; x^{+}-, x^{\prime+} \alpha^{\prime}\right) .
\end{aligned}
$$

By adding and subtracting both sides of the above equations, we obtain:

$$
\left\{\begin{array}{rl}
-i \sum_{\alpha^{\prime}, \beta^{\prime}} \sigma_{\alpha^{\prime}, \beta^{\prime}}^{\nu} L\left(x+, x^{\prime} \beta^{\prime} ; x^{+}+, x^{+} \alpha^{\prime}\right) & =2\left[\mathcal{X}_{0, \nu}\left(x, x^{\prime}\right)+\mathcal{X}_{z, \nu}\left(x, x^{\prime}\right)\right] \\
-i \sum_{\alpha^{\prime}, \beta^{\prime}} \sigma_{\alpha^{\prime}, \beta^{\prime}}^{\nu} L\left(x-, x^{\prime} \beta^{\prime} ; x^{+}-, x^{\prime+} \alpha^{\prime}\right) & =2\left[\mathcal{X}_{0, \nu}\left(x, x^{\prime}\right)-\mathcal{X}_{z, \nu}\left(x, x^{\prime}\right)\right]
\end{array} .\right.
$$

For $\mu=x$ and $\mu=y$ we obtain instead:

$$
\left\{\begin{array}{l}
-i \sum_{\alpha^{\prime}, \beta^{\prime}} \sigma_{\alpha^{\prime}, \beta^{\prime}}^{\nu} L\left(x-, x^{\prime} \beta^{\prime} ; x^{+}+, x^{+} \alpha^{\prime}\right)=2\left[\mathcal{X}_{x, \nu}\left(x, x^{\prime}\right)+i \mathcal{X}_{y, \nu}\left(x, x^{\prime}\right)\right] \\
-i \sum_{\alpha^{\prime}, \beta^{\prime}}^{\prime} \sigma_{\alpha^{\prime}, \beta^{\prime}}^{\nu} L\left(x+, x^{\prime} \beta^{\prime} ; x^{+}-, x^{\prime+} \alpha^{\prime}\right)=2\left[\mathcal{X}_{x, \nu}\left(x, x^{\prime}\right)-i \mathcal{X}_{y, \nu}\left(x, x^{\prime}\right)\right]
\end{array},\right.
$$

with similar relations holding for the non-interacting counterparts of $L$ and $\mathcal{X}$. Entering these relations into Eq. (10), the closed-form expression (11) results eventually after suitable manipulation.

Next, we specify the form of the non-interacting counterpart $\mathcal{X}^{(0)}$ of $\mathcal{X}$, as defined by the first term on the right-hand side of Eq. (10), namely: 


$$
\begin{aligned}
\mathcal{X}_{\mu, \nu}^{(0)}\left(x, x^{\prime}\right) & \equiv-\frac{i}{4} \sum_{\alpha, \beta} \sum_{\alpha^{\prime}, \beta^{\prime}} \sigma_{\alpha, \beta}^{\mu} \sigma_{\alpha^{\prime}, \beta^{\prime}}^{\nu} G\left(x \beta, x^{\prime} \alpha^{\prime}\right) G\left(x^{\prime} \beta^{\prime}, x \alpha\right) \\
& =\frac{i}{4} \sum_{\alpha, \beta} \sum_{\alpha^{\prime}, \beta^{\prime}} \sigma_{\alpha, \beta}^{\mu} \sigma_{\alpha^{\prime}, \beta^{\prime}}^{\nu}\left\langle T\left[\psi_{\beta}(x) \psi_{\alpha^{\prime}}^{\dagger}\left(x^{\prime}\right)\right]\right\rangle\left\langle T\left[\psi_{\beta^{\prime}}\left(x^{\prime}\right) \psi_{\alpha}^{\dagger}(x)\right]\right\rangle .
\end{aligned}
$$

To this end, we follow Ref 16 and introduce the set of destruction operators $d_{i \xi}$ along the local spin-quantization axes, which identify the spiral pattern of the magnetic ground state within the mean-field approximation. The field operator (3) acquires then the form:

$$
\begin{aligned}
\psi_{\alpha}(\mathbf{r}) & =\sum_{i} \sum_{\xi} \phi\left(\mathbf{r}-\mathbf{R}_{i}\right) \mathcal{R}\left(\Omega_{i}\right)_{\alpha \xi} d_{i \xi} \\
& =\sum_{i} \sum_{\xi} \sum_{\mathbf{k}}^{B Z} \phi\left(\mathbf{r}-\mathbf{R}_{i}\right) \mathcal{R}\left(\Omega_{i}\right)_{\alpha \xi} \frac{\mathrm{e}^{i \mathbf{k} \cdot \mathbf{R}_{i}}}{\sqrt{\mathcal{N}}} d_{\mathbf{k} \xi}
\end{aligned}
$$

where $\mathcal{R}\left(\Omega_{i}\right)$ is the spin rotation operator associated with the angles $\Omega_{i}$ and where the Bloch transformation has been introduced as in Section 3, which brings the mean-field Hubbard Hamiltonian into block form for each wave vector $\mathbf{k}$ belonging to the Brillouin zone $(B Z)$.

Let $W_{\xi, r}(\mathbf{k})$ (with $\xi=+,-$ ) be the matrix which diagonalizes the mean-field Hubbard Hamiltonian at given $\mathbf{k}$, such that [cf. Eq. (26)]

$$
\sum_{\mathbf{k}}^{B Z} \sum_{\xi, \xi^{\prime}} d_{\mathbf{k} \xi}^{\dagger} \mathcal{H}_{\xi, \xi^{\prime}}(\mathbf{k}) d_{\mathbf{k} \xi^{\prime}}=\sum_{\mathbf{k}}^{B Z} \sum_{r} \gamma_{\mathbf{k}, r}^{\dagger} \epsilon_{r}(\mathbf{k}) \gamma_{\mathbf{k}, r}
$$

with

$$
\gamma_{\mathbf{k}, r}=\sum_{\xi} W_{r, \xi}^{\dagger}(\mathbf{k}) d_{\mathbf{k} \xi}
$$

and $\epsilon_{r}(\mathbf{k})$ given by Eq. (29). Upon averaging over the the broken-symmetry ground state, one then obtains:

$$
\left\langle T\left[d_{\mathbf{k} \xi}(t) d_{\mathbf{k}^{\prime} \xi^{\prime}}^{\dagger}\left(t^{\prime}\right)\right]\right\rangle=\sum_{r, r^{\prime}} W_{\xi, r}(\mathbf{k}) W_{r^{\prime}, \xi^{\prime}}^{\dagger}\left(\mathbf{k}^{\prime}\right)\left\langle T\left[\gamma_{\mathbf{k} r}(t) \gamma_{\mathbf{k}^{\prime} r^{\prime}}^{\dagger}\left(t^{\prime}\right)\right]\right\rangle
$$

where

$$
\begin{aligned}
& \left\langle T\left[\gamma_{\mathbf{k} r}(t) \gamma_{\mathbf{k}^{\prime} r^{\prime}}^{\dagger}\left(t^{\prime}\right)\right]\right\rangle \\
& =\delta_{\mathbf{k}, \mathbf{k}^{\prime}} \delta_{r, r^{\prime}} e^{-i \epsilon_{r}(\mathbf{k})\left(t-t^{\prime}\right)}\left\{\Theta\left(t-t^{\prime}\right)\left[1-f_{F}\left(\epsilon_{r}(\mathbf{k})\right)\right]-\Theta\left(t^{\prime}-t\right) f_{F}\left(\epsilon_{r}(\mathbf{k})\right)\right\}
\end{aligned}
$$

$\Theta(t)$ being the unit step function. Introducing further the tensor $T\left(\Omega_{i}\right)$ via the relation

$$
\sum_{\alpha, \beta} \mathcal{R}^{\dagger}\left(\Omega_{i}\right)_{\xi \alpha} \sigma_{\alpha, \beta}^{\mu} \mathcal{R}\left(\Omega_{i}\right)_{\beta, \xi^{\prime}}=\sum_{\nu} T_{\mu \nu}\left(\Omega_{i}\right) \sigma_{\xi, \xi^{\prime}}^{\nu}
$$

such that

$$
T\left(\Omega_{i}\right)=\left(\begin{array}{cccc}
1 & 0 & 0 & 0 \\
0 & \cos \theta_{i} & 0 & \sin \theta_{i} \\
0 & 0 & 1 & 0 \\
0 & -\sin \theta_{i} & 0 & \cos \theta_{i}
\end{array}\right)
$$

where $\theta_{i}=\mathbf{Q} \cdot \mathbf{R}_{i}$ within the spiral-spin pattern we are considering, 16 and approximating

$$
\phi^{*}\left(\mathbf{r}-\mathbf{R}_{i}\right) \phi\left(\mathbf{r}-\mathbf{R}_{j}\right) \cong\left|\phi\left(\mathbf{r}-\mathbf{R}_{i}\right)\right|^{2} \delta_{i, j}
$$

for localized atomic (Wannier) orbitals, we obtain the following expression for the frequency Fourier transform of the non-interacting correlation function: 


$$
\begin{aligned}
\mathcal{X}_{\mu, \nu}^{(0)}\left(\mathbf{r}, \mathbf{r}^{\prime} ; \omega\right) & =\frac{1}{4 \mathcal{N}^{2}} \sum_{i, j} \sum_{\mathbf{k}, \mathbf{k}^{\prime}}^{B Z} e^{i\left(\mathbf{k}-\mathbf{k}^{\prime}\right) \cdot\left(\mathbf{R}_{i}-\mathbf{R}_{j}\right)}\left|\phi\left(\mathbf{r}-\mathbf{R}_{i}\right)\right|^{2}\left|\phi\left(\mathbf{r}^{\prime}-\mathbf{R}_{j}\right)\right|^{2} \\
& \times \sum_{r, r^{\prime}} \sum_{\mu^{\prime}, \nu^{\prime}} T_{\mu, \mu^{\prime}}\left(\Omega_{i}\right) T_{\nu, \nu^{\prime}}\left(\Omega_{j}\right) F_{r^{\prime}, r}^{\mu^{\prime}}\left(\mathbf{k}^{\prime}, \mathbf{k}\right) F_{r, r^{\prime}}^{\nu^{\prime}}\left(\mathbf{k}, \mathbf{k}^{\prime}\right) \\
& \times \mathcal{F}_{r, r^{\prime}}\left(\mathbf{k}, \mathbf{k}^{\prime}, \omega\right) .
\end{aligned}
$$

Here we have introduced the notation:

$$
F_{r, r^{\prime}}^{\mu}\left(\mathbf{k}, \mathbf{k}^{\prime}\right) \equiv \sum_{\xi, \xi^{\prime}} W_{r, \xi}^{\dagger}(\mathbf{k}) \sigma_{\xi, \xi^{\prime}}^{\mu} W_{\xi^{\prime}, r^{\prime}}\left(\mathbf{k}^{\prime}\right)
$$

as well as

$$
\begin{aligned}
\mathcal{F}_{r, r^{\prime}}\left(\mathbf{k}, \mathbf{k}^{\prime}, \omega\right)= & \frac{\left[1-f_{F}\left(\epsilon_{r}(\mathbf{k})\right)\right] f_{F}\left(\epsilon_{r^{\prime}}\left(\mathbf{k}^{\prime}\right)\right)}{\omega-\epsilon_{r}(\mathbf{k})+\epsilon_{r^{\prime}}\left(\mathbf{k}^{\prime}\right)+i \eta} \\
- & \frac{\left[1-f_{F}\left(\epsilon_{r^{\prime}}\left(\mathbf{k}^{\prime}\right)\right)\right] f_{F}\left(\epsilon_{r}(\mathbf{k})\right)}{\omega-\epsilon_{r}(\mathbf{k})+\epsilon_{r^{\prime}}\left(\mathbf{k}^{\prime}\right)-i \eta}
\end{aligned}
$$

where $f_{F}(\epsilon)$ is the Fermi function. Equation (13) of the text is thus recovered.

We have mentioned in Section 2 that the solution to the integral equation (11) could be considerably simplified, provided the matrix $T\left(\Omega_{i}\right)$ given by Eq. A12) were preliminary brought to diagonal form by a suitable unitary transformation. This transformation reads:

$$
\bar{\sigma}^{a}=\sum_{\mu=(0, x, y, z)} B_{a \mu} \sigma^{\mu}
$$

with $a=(0,1,2,3)$ and where

$$
B=\left(\begin{array}{cccc}
1 & 0 & 0 & 0 \\
0 & \frac{1}{\sqrt{2}} & 0 & \frac{i}{\sqrt{2}} \\
0 & 0 & 1 & 0 \\
0 & \frac{1}{\sqrt{2}} & 0 & \frac{-i}{\sqrt{2}}
\end{array}\right)
$$

In this way, Eq. $\mathrm{A15}$ is replaced by:

$$
\bar{F}_{r, r^{\prime}}^{a}\left(\mathbf{k}, \mathbf{k}^{\prime}\right)=\sum_{\mu=(0, x, y, z)} B_{a \mu} F_{r, r^{\prime}}^{\mu}\left(\mathbf{k}, \mathbf{k}^{\prime}\right),
$$

and the correlation functions transform according to the rule:

$$
\overline{\mathcal{X}}_{a b}=\sum_{\mu, \nu} B_{a \mu} \mathcal{X}_{\mu \nu}\left(B^{T}\right)_{\nu b} .
$$

The second term on the right-hand side of the integral equation (11) then transforms as follows:

$$
B \mathcal{X}^{(0)} \epsilon \mathcal{X} B^{T}=B \mathcal{X}^{(0)} B^{T}\left(B^{T}\right)^{-1} \epsilon B^{-1} B \mathcal{X} B^{T}=\overline{\mathcal{X}}^{(0)} \bar{\epsilon} \overline{\mathcal{X}}
$$

in matrix notation, where

$$
\bar{\epsilon}=\left(B^{T}\right)^{-1} \epsilon B^{-1}=\left(\begin{array}{rrrr}
1 & 0 & 0 & 0 \\
0 & 0 & 0 & -1 \\
0 & 0 & -1 & 0 \\
0 & -1 & 0 & 0
\end{array}\right)
$$

at the same time

$$
\bar{T}\left(\Omega_{i}\right)=B T\left(\Omega_{i}\right) B^{-1}=\left(\begin{array}{cccc}
1 & 0 & 0 & 0 \\
0 & e^{-i \mathbf{Q} \cdot \mathbf{R}_{i}} & 0 & 0 \\
0 & 0 & 1 & 0 \\
0 & 0 & 0 & e^{i \mathbf{Q} \cdot \mathbf{R}_{i}}
\end{array}\right)
$$


becomes diagonal, as anticipated. Equation (16) of the text then follows.

There remains to solve Eq. (18) of the text explicitly. To this end, we introduce the compact notation

$$
\gamma_{a}=\left\{\begin{aligned}
0 & (a=0) \\
-1 & (a=1) \\
0 & (a=2) \\
+1 & (a=3)
\end{aligned}\right.
$$

in such a way that (the lattice Fourier transform of) Eq. (A23) reads:

$$
\bar{T}_{a a^{\prime}}(\mathbf{k})=\delta_{a, a^{\prime}} \Delta\left(\mathbf{k}+\gamma_{a} \mathbf{Q}\right)
$$

$\Delta(\mathbf{k})$ being the lattice Kronecker delta function. In the transformed basis (cf. Eq. (A20)), the non-interacting part (17) of the correlation function then takes the form:

$$
\hat{\mathcal{X}}_{a b}^{(0)}\left(\mathbf{q}, \mathbf{q}^{\prime} ; \omega\right)=\Delta\left(\mathbf{q}-\mathbf{q}^{\prime}-\left(\gamma_{a}+\gamma_{b}\right) \mathbf{Q}\right) X_{a b}^{(0)}(\mathbf{q} ; \omega \mid \mathbf{Q})
$$

where we have set

$$
\begin{aligned}
X_{a b}^{(0)}(\mathbf{q} ; \omega \mid \mathbf{Q}) & =\frac{1}{4 \mathcal{N}} \sum_{\mathbf{k}}^{B Z} \sum_{r, r^{\prime}} \bar{F}_{r^{\prime}, r}^{a}\left(\mathbf{k}-\mathbf{q}+\gamma_{a} \mathbf{Q}, \mathbf{k}\right) \bar{F}_{r, r^{\prime}}^{b}\left(\mathbf{k}, \mathbf{k}-\mathbf{q}+\gamma_{a} \mathbf{Q}\right) \\
& \times \mathcal{F}_{r r^{\prime}}\left(\mathbf{k}, \mathbf{k}-\mathbf{q}+\gamma_{a} \mathbf{Q}\right) .
\end{aligned}
$$

Owing to the wave vector conserving Kronecker delta function in Eq. (A26), the integral equation (18) becomes:

$$
\begin{aligned}
\hat{\mathcal{X}}_{a b}\left(\mathbf{q}, \mathbf{q}^{\prime} ; \omega\right) & =\Delta\left(\mathbf{q}-\mathbf{q}^{\prime}-\left(\gamma_{a}+\gamma_{b}\right) \mathbf{Q}\right) X_{a b}^{(0)}(\mathbf{q} ; \omega \mid \mathbf{Q}) \\
& +2 U\left[X_{a 0}^{(0)}(\mathbf{q} ; \omega \mid \mathbf{Q}) \hat{\mathcal{X}}_{0 b}\left(\mathbf{q}-\gamma_{a} \mathbf{Q}, \mathbf{q}^{\prime} ; \omega\right)\right. \\
& -X_{a 1}^{(0)}(\mathbf{q} ; \omega \mid \mathbf{Q}) \hat{\mathcal{X}}_{3 b}\left(\mathbf{q}-\left(\gamma_{a}-1\right) \mathbf{Q}, \mathbf{q}^{\prime} ; \omega\right) \\
& -X_{a 2}^{(0)}(\mathbf{q} ; \omega \mid \mathbf{Q}) \hat{\mathcal{X}}_{2 b}\left(\mathbf{q}-\gamma_{a} \mathbf{Q}, \mathbf{q}^{\prime} ; \omega\right) \\
& \left.-X_{a 3}^{(0)}(\mathbf{q} ; \omega \mid \mathbf{Q}) \hat{\mathcal{X}}_{1 b}\left(\mathbf{q}-\left(\gamma_{a}+1\right) \mathbf{Q}, \mathbf{q}^{\prime} ; \omega\right)\right] .
\end{aligned}
$$

Note that the wave vector arguments on the right-hand side of the above expression depend on the index $a$ of the matrix element. To avoid this feature, we let $\mathbf{q} \rightarrow \mathbf{q}+\gamma_{a} \mathbf{Q}$ everywhere in the above expression and obtain:

$$
\begin{aligned}
\hat{\mathcal{X}}_{a b}\left(\mathbf{q}+\gamma_{a} \mathbf{Q}, \mathbf{q}^{\prime} ; \omega\right) & =\Delta\left(\mathbf{q}-\mathbf{q}^{\prime}-\gamma_{b} \mathbf{Q}\right) X_{a b}^{(0)}\left(\mathbf{q}+\gamma_{a} \mathbf{Q} ; \omega \mid \mathbf{Q}\right) \\
& +2 U\left[X_{a 0}^{(0)}\left(\mathbf{q}+\gamma_{a} \mathbf{Q} ; \omega \mid \mathbf{Q}\right) \hat{\mathcal{X}}_{0 b}\left(\mathbf{q}, \mathbf{q}^{\prime} ; \omega\right)\right. \\
& -X_{a 1}^{(0)}\left(\mathbf{q}+\gamma_{a} \mathbf{Q} ; \omega \mid \mathbf{Q}\right) \hat{\mathcal{X}}_{3 b}\left(\mathbf{q}+\mathbf{Q}, \mathbf{q}^{\prime} ; \omega\right) \\
& -X_{a 2}^{(0)}\left(\mathbf{q}+\gamma_{a} \mathbf{Q} ; \omega \mid \mathbf{Q}\right) \hat{\mathcal{X}}_{2 b}\left(\mathbf{q}, \mathbf{q}^{\prime} ; \omega\right) \\
& \left.-X_{a 3}^{(0)}\left(\mathbf{q}+\gamma_{a} \mathbf{Q} ; \omega \mid \mathbf{Q}\right) \hat{\mathcal{X}}_{1 b}\left(\mathbf{q}-\mathbf{Q}, \mathbf{Q}^{\prime} ; \omega\right)\right] .
\end{aligned}
$$

For any given value of the index $b$, this relation can then be cast in the equivalent form:

$$
[\mathbf{1}+X(\mathbf{q}, \omega)]\left(\begin{array}{c}
\hat{\mathcal{X}}_{0 b}\left(\mathbf{q}, \mathbf{q}^{\prime} ; \omega\right) \\
\hat{\mathcal{X}}_{1 b}\left(\mathbf{q}-\mathbf{Q}, \mathbf{q}^{\prime} ; \omega\right) \\
\hat{\mathcal{X}}_{2 b}\left(\mathbf{q}, \mathbf{q}^{\prime} ; \omega\right) \\
\hat{\mathcal{X}}_{3 b}\left(\mathbf{q}+\mathbf{Q}, \mathbf{q}^{\prime} ; \omega\right)
\end{array}\right)=\left(\begin{array}{c}
X_{0 b}^{(0)}(\mathbf{q} ; \omega \mid \mathbf{Q}) \\
X_{1 b}^{(0)}(\mathbf{q}-\mathbf{Q} ; \omega \mid \mathbf{Q}) \\
X_{2 b}^{(0)}(\mathbf{q} ; \omega \mid \mathbf{Q}) \\
X_{3 b}^{(0)}(\mathbf{q}+\mathbf{Q} ; \omega \mid \mathbf{Q})
\end{array}\right) \Delta\left(\mathbf{q}-\mathbf{q}^{\prime}-\gamma_{b} \mathbf{Q}\right)
$$

where we have set

$$
\begin{aligned}
& X(\mathbf{q}, \omega)=2 U \\
& \times\left(\begin{array}{cccc}
-X_{00}^{(0)}(\mathbf{q} ; \omega \mid \mathbf{Q}) & X_{03}^{(0)}(\mathbf{q} ; \omega \mid \mathbf{Q}) & X_{02}^{(0)}(\mathbf{q} ; \omega \mid \mathbf{Q}) & X_{01}^{(0)}(\mathbf{q} ; \omega \mid \mathbf{Q}) \\
-X_{10}^{(0)}(\mathbf{q}-\mathbf{Q} ; \omega \mid \mathbf{Q}) & X_{13}^{(0)}(\mathbf{q}-\mathbf{Q} ; \omega \mid \mathbf{Q}) & X_{12}^{(0)}(\mathbf{q}-\mathbf{Q} ; \omega \mid \mathbf{Q}) & X_{11}^{(0)}(\mathbf{q}-\mathbf{Q} ; \omega \mid \mathbf{Q}) \\
-X_{20}^{(0)}(\mathbf{q} ; \omega \mid \mathbf{Q}) & X_{23}^{(0)}(\mathbf{Q} ; \omega \mid \mathbf{Q}) & X_{22}^{(0)}(\mathbf{q} ; \omega \mid \mathbf{Q}) & X_{21}^{(0)}(\mathbf{Q} ; \omega \mid \mathbf{Q}) \\
-X_{30}^{(0)}(\mathbf{q}+\mathbf{Q} ; \omega \mid \mathbf{Q}) & X_{33}^{(0)}(\mathbf{q}+\mathbf{Q} ; \omega \mid \mathbf{Q}) & X_{32}^{(0)}(\mathbf{q}+\mathbf{Q} ; \omega \mid \mathbf{Q}) & X_{31}^{(0)}(\mathbf{Q}+\mathbf{Q} ; \omega \mid \mathbf{Q})
\end{array}\right) .
\end{aligned}
$$


Solving for $\hat{\mathcal{X}}_{a b}$ in Eq. (A29), we obtain eventually:

$$
\begin{aligned}
\hat{\mathcal{X}}_{a b}\left(\mathbf{q}+\gamma_{a} \mathbf{Q}, \mathbf{q}^{\prime} ; \omega\right) & =\sum_{a^{\prime}}[\mathbf{1}+X(\mathbf{q}, \omega)]_{a a^{\prime}}^{-1} \\
& \times X_{a^{\prime} b}^{(0)}\left(\mathbf{q}+\gamma_{a^{\prime}} \mathbf{Q} ; \omega \mid \mathbf{Q}\right) \Delta\left(\mathbf{q}-\mathbf{q}^{\prime}-\gamma_{b} \mathbf{Q}\right)
\end{aligned}
$$

which coincides with Eq. (19) of the text.

\section{APPENDIX B: DETAILS OF THE $t / U$ EXPANSION}

In this Appendix, we provide details of the $t / U$ expansion of the mean-field parameters and of the matrix elements of the correlation function which are necessary to obtain the spin-wave dispersion relation.

We begin by considering the leading $(t=0)$ term of the expansion (35) for the mean-field (band) eigenvalues, corresponding to completely flat bands, which is given by [cf. Eq. (29)]

$$
\epsilon_{r}(\mathbf{k})=U\left(\epsilon_{r}^{(0)}-\mu^{(0)}\right)=U\left(m_{1}+(-1)^{r} m_{2}\right)-\mu_{0}
$$

with $r=1,2$ and where $\mu_{0}=U \mu^{(0)}$ is the chemical potential at the lowest order in $t / U$. Since at this order the band eigenvalues do not depend on the wave vector, there is no value of the chemical potential $\mu^{(0)}$ satisfying equation (32) at zero temperature and noninteger doping $\delta$. More precisely, the doping jumps from $\delta=-1$ when $\mu^{(0)}<\epsilon_{1}^{(0)}$, to $\delta=0$ when $\epsilon_{1}^{(0)}<\mu^{(0)}<\epsilon_{2}^{(0)}$, and to $\delta=+1$ when $\mu^{(0)}>\epsilon_{2}^{(0)}$. In order to consider a continuously doped system, it is therefore necessary to include at the outset the next-to-leading order in the expansion (35) of the band eigenvalues, which introduces a band dispersion.

For definiteness, we shall consider the case $\delta>0$ from now on. (The case $\delta<0$ can be recovered by exploiting particle-hole symmetry.) In the case $\delta>0$, the lowest band is always filled, and we will consistently use (the zerotemperature value) $f_{F}\left(\epsilon_{1}(\mathbf{k})\right)=1$ throughout. On the other hand, the zeroth order chemical potential for $0<\delta<1$ must be chosen as $\mu^{(0)}=\epsilon_{2}^{(0)}$, and the Fermi function in the upper band can be expanded as

$$
\begin{aligned}
& f_{F}\left(\epsilon_{2}(\mathbf{k})\right)=1-\Theta\left(\epsilon_{2}^{(1)}(\mathbf{k})-\mu^{(1)}\right) \\
& -\delta\left(\epsilon_{2}^{(1)}(\mathbf{k})-\mu^{(1)}\right)\left[\left(\frac{t}{U}\right)\left(\epsilon_{2}^{(2)}(\mathbf{k})-\mu^{(2)}\right)+\left(\frac{t}{U}\right)^{2}\left(\epsilon_{2}^{(3)}(\mathbf{k})-\mu^{(3)}\right)\right] \\
& -\frac{1}{2} \delta^{\prime}\left(\epsilon_{2}^{(1)}(\mathbf{k})-\mu^{(1)}\right)\left(\frac{t}{U}\right)^{2}\left(\epsilon_{2}^{(2)}(\mathbf{k})-\mu^{(2)}\right)^{2}+\cdots,
\end{aligned}
$$

where we have considered the zero-temperature limit of the Fermi function and of its derivatives, and introduced the step function $\Theta$ as well as the Dirac $\delta(x)$ function (not to be confused with doping). In this way, Eq. (32) can be expressed in powers of $t / U$, yielding

$$
\begin{aligned}
& \frac{1}{\mathcal{N}} \sum_{\mathbf{k}}^{B Z} \Theta\left(\epsilon_{2}^{(1)}(\mathbf{k})-\mu^{(1)}\right)=1-\delta \\
& \frac{1}{\mathcal{N}} \sum_{\mathbf{k}}^{B Z} \delta\left(\epsilon_{2}^{(1)}(\mathbf{k})-\mu^{(1)}\right)\left(\epsilon_{2}^{(2)}(\mathbf{k})-\mu^{(2)}\right)=0 \\
& \frac{1}{2 \mathcal{N}} \sum_{\mathbf{k}}^{B Z}\left[\delta^{\prime}\left(\epsilon_{2}^{(1)}(\mathbf{k})-\mu^{(1)}\right)\left(\epsilon_{2}^{(2)}(\mathbf{k})-\mu^{(2)}\right)^{2}+2 \delta\left(\epsilon_{2}^{(1)}(\mathbf{k})-\mu^{(1)}\right)\left(\epsilon_{2}^{(3)}(\mathbf{k})-\mu^{(3)}\right)\right]=0 .
\end{aligned}
$$

Similarly, the small $t / U$ expansion (36) of Eq. (33) yields [cf. also Eqs. (37)-(39)]:

$$
\begin{aligned}
& m_{2}^{(0)}+\left(\frac{t}{U}\right) m_{2}^{(1)}+\left(\frac{t}{U}\right)^{2} m_{2}^{(2)}+\cdots \\
& =\frac{1}{2 \mathcal{N}} \sum_{\mathbf{k}}^{B Z}\left[1-2\left(\frac{t}{U}\right)^{2}\left(\frac{T_{o}(\mathbf{k})}{2 m_{2}^{(0)}}\right)^{2}\right]\left(1-f_{F}\left(\epsilon_{2}(\mathbf{k})\right)\right)+\mathcal{O}\left(\left(\frac{U}{t}\right)^{3}\right),
\end{aligned}
$$


where the Fermi function should be again expanded in the form (B2). One obtains eventually:

$$
\begin{aligned}
m_{2}^{(0)} & =\frac{1}{2 \mathcal{N}} \sum_{\mathbf{k}}^{B Z} \Theta\left(\epsilon_{2}^{(1)}(\mathbf{k})-\mu^{(1)}\right), \\
m_{2}^{(1)} & =\frac{1}{2 \mathcal{N}} \sum_{\mathbf{k}}^{B Z} \delta\left(\epsilon_{2}^{(1)}(\mathbf{k})-\mu^{(1)}\right)\left(\epsilon_{2}^{(2)}(\mathbf{k})-\mu^{(2)}\right), \\
m_{2}^{(2)} & =-\frac{1}{\mathcal{N}} \sum_{\mathbf{k}}^{B Z}\left(\frac{T_{o}(\mathbf{k})}{2 m_{2}^{(0)}}\right)^{2} \Theta\left(\epsilon_{2}^{(1)}(\mathbf{k})-\mu^{(1)}\right) \\
& +\frac{1}{4 \mathcal{N}} \sum_{\mathbf{k}}^{B Z} \delta^{\prime}\left(\epsilon_{2}^{(1)}(\mathbf{k})-\mu^{(1)}\right)\left(\epsilon_{2}^{(2)}(\mathbf{k})-\mu^{(2)}\right)^{2} \\
& +\frac{1}{2 \mathcal{N}} \sum_{\mathbf{k} Z}^{B Z} \delta\left(\epsilon_{2}^{(1)}(\mathbf{k})-\mu^{(1)}\right)\left(\epsilon_{2}^{(3)}(\mathbf{k})-\mu^{(3)}\right) \\
& =-\frac{1}{\mathcal{N}} \sum_{\mathbf{k}}^{B Z}\left(\frac{T_{o}(\mathbf{k})}{2 m_{2}^{(0)}}\right)^{2} \Theta\left(\epsilon_{2}^{(1)}(\mathbf{k})-\mu^{(1)}\right),
\end{aligned}
$$

where in the last step we have used the last of Eqs. (B3).

There remains to expand in powers of $t / U$ the last the self-consistency equation (34). For the diagonal spin-spiral solution we obtain:

$$
\begin{aligned}
& 0=\sin (Q / 2) \frac{1}{\mathcal{N}} \sum_{\mathbf{k}}^{B Z}\left(\cos k_{x}+\cos k_{y}\right)\left(1+f_{F}\left(\epsilon_{2}(\mathbf{k})\right)\right) \\
& +\left(\frac{t}{U}\right) \cos (Q / 2) \frac{1}{m_{2}^{(0)}} \frac{1}{\mathcal{N}} \sum_{\mathbf{k}}^{B Z} T_{o}(\mathbf{k})\left(\sin k_{x}+\sin k_{y}\right)\left(1-f_{F}\left(\epsilon_{2}(\mathbf{k})\right)\right)
\end{aligned}
$$

where the expansion (B2) has still to be inserted.

From Eq. (29), the argument of the $\Theta$ function is $T_{e}(\mathbf{k})+m_{2}^{(1)}-\mu^{(1)}\left(\right.$ with $T_{e}(\mathbf{k})=2 \cos (Q / 2)\left(\cos k_{x}+\cos k_{y}\right)$ for the diagonal spiral solution), which can take positive as well as negative values.

At the various orders in $t / U$ we then obtain from Eqs. (B3) and (B5)):

(i) order $(t / U)^{0}$

$$
\begin{aligned}
& \frac{1}{\mathcal{N}} \sum_{\mathbf{k}}^{B Z}\left[1-\Theta\left(T_{e}(\mathbf{k})+m_{2}^{(1)}-\mu^{(1)}\right)\right]=\delta, \\
& m_{2}^{(0)}=\frac{1}{2 \mathcal{N}} \sum_{\mathbf{k}}^{B Z} \Theta\left(T_{e}(\mathbf{k})+m_{2}^{(1)}-\mu^{(1)}\right) ;
\end{aligned}
$$

(ii) order $(t / U)^{1}$

$$
\begin{aligned}
& \frac{1}{\mathcal{N}} \sum_{\mathbf{k}}^{B Z} \delta\left(T_{e}(\mathbf{k})+m_{2}^{(1)}-\mu^{(1)}\right)\left(\epsilon_{2}^{(2)}(\mathbf{k})-\mu^{(2)}\right)=0 \\
& m_{2}^{(1)}=\frac{1}{2 \mathcal{N}} \sum_{\mathbf{k}}^{B Z} \delta\left(T_{e}(\mathbf{k})+m_{2}^{(1)}-\mu^{(1)}\right)\left(\epsilon_{2}^{(2)}(\mathbf{k})-\mu^{(2)}\right)
\end{aligned}
$$

(iii) order $(t / U)^{2}$

$$
m_{2}^{(2)}=-\frac{1}{\mathcal{N}} \sum_{\mathbf{k}}^{B Z}\left(\frac{T_{o}(\mathbf{k})}{2 m_{2}^{(0)}}\right)^{2} \Theta\left(T_{e}(\mathbf{k})+m_{2}^{(1)}-\mu^{(1)}\right)
$$


and we don't need to evaluate $\left(\epsilon_{2}^{(3)}(\mathbf{k})-\mu^{(3)}\right)$ at the order we are considering. It is convenient to solve these equations order-by-order for the two variables $\mu^{(i)}-m_{2}^{(i)}$, and $m_{2}^{(i)}(i=0,1,2)$.

Using at this point the method developed in Appendix $\mathrm{C}$ for performing the $\mathbf{k}$ summation over the relevant portions of the Brillouin zone, we obtain eventually the following results for the mean-field parameters at the leading orders in $\delta$ :

$$
\left\{\begin{array}{l}
m_{2}^{(0)}=(1-\delta) / 2 \\
m_{2}^{(1)}=0 \\
m_{2}^{(2)}=-4 \sin ^{2}(Q / 2)+\mathcal{O}(\delta)
\end{array}\right.
$$

and

$$
\left\{\begin{array}{l}
\mu^{(0)}-m_{2}^{(0)}=m_{1}=(1+\delta) / 2 \\
\mu^{(1)}-m_{2}^{(1)}=4 \cos (Q / 2)\left(1-\pi \delta+\mathcal{O}\left(\delta^{2}\right)\right) \\
\mu^{(2)}-m_{2}^{(2)}=0+\mathcal{O}(\delta) .
\end{array}\right.
$$

There remains to determine the magnitude $Q$ of the characteristic wave vector from the self-consistency condition (B6), where for the Fermi function we use the expansion (B2). Equation (B6) then becomes:

$$
\begin{aligned}
& \left(\frac{t}{U}\right) \sin (Q / 2) \frac{1}{\mathcal{N}} \sum_{\mathbf{k}}^{B Z}\left(\cos k_{x}+\cos k_{y}\right)\left[2-\Theta\left(T_{e}(\mathbf{k})+m_{2}^{(1)}-\mu^{(1)}\right)\right. \\
& \left.-\left(\frac{t}{U}\right) \delta\left(T_{e}(\mathbf{k})+m_{2}^{(1)}-\mu^{(1)}\right)\left(\epsilon_{2}^{(2)}(\mathbf{k})-\mu^{(2)}\right)\right]+2\left(\frac{t}{U}\right)^{2} \cos (Q / 2) \\
& \times \frac{1}{2 m_{2}^{(0)}} \frac{1}{\mathcal{N}} \sum_{\mathbf{k}}^{B Z}\left(\sin k_{x}+\sin k_{y}\right) T_{o}(\mathbf{k}) \Theta\left(T_{e}(\mathbf{k})+m_{2}^{(1)}-\mu^{(1)}\right) \\
& =0 .
\end{aligned}
$$

In particular, at the lowest order in $t / U$ we obtain:

$$
\sin (Q / 2)\left(2 \delta+\mathcal{O}\left(\delta^{2}\right)\right)=0 .
$$

For fixed $\delta$ and large $U / t$, one thus has only the ferromagnetic solution $Q=0$. However, if one allows $\delta$ to be of the order $t / U$, Eq. (B13) has to be considered together with the next term in $t / U$, and we obtain instead:

$$
\sin (Q / 2)\left(2 \delta+\mathcal{O}\left(\delta^{2}\right)\right)+4 \sin (Q / 2) \cos (Q / 2)(1+\mathcal{O}(\delta))=0
$$

which yields the two solutions

$$
\begin{array}{cc}
\sin (Q / 2)=0 & \text { (ferromagnet) } \\
\cos (Q / 2)=-\delta U / 2 t \quad \text { (diagonal spiral) },
\end{array}
$$

with the spiral solution being energetically favored over the ferromagnetic solution whenever it exists. From the above equation, it is clear that the spiral solution exists for any $\delta \leq 2 t / U$, which is consistent with our assumption that the doping parameter $\delta$ is at most of order $t / U$ in the spiral phase, thus justifying the expansion of Appendix C. The transition to the ferromagnetic state is second order, as the incommensurability vector $Q$ decreases continuously from $Q=\pi$ to $Q=2 \pi$ with increasing $\delta$.

We pass finally to consider the $t / U$ expansion of the matrix elements of the correlation function. To begin with, we use the expressions (37)-(39) for the eigenvectors of the mean-field Hamiltonian and obtain from the definitions (22) and $(23)$ :

$$
\begin{aligned}
\mathcal{X}_{0}^{+,-}(\mathbf{q}, \omega) & =\frac{1}{2 \mathcal{N}} \sum_{\mathbf{k}}^{B Z}\left\{\left[1-\left(\frac{t}{U}\right)^{2}\left(T_{o}^{2}(\mathbf{k})+T_{o}^{2}(\mathbf{k}-\mathbf{q})\right)\right] \mathcal{F}_{2,1}(\mathbf{k}, \mathbf{k}-\mathbf{q}, \omega)\right. \\
& \left.+\left(\frac{t}{U}\right)^{2}\left[T_{o}^{2}(\mathbf{k}) \mathcal{F}_{1,1}(\mathbf{k}, \mathbf{k}-\mathbf{q}, \omega)+T_{o}^{2}(\mathbf{k}-\mathbf{q}) \mathcal{F}_{2,2}(\mathbf{k}, \mathbf{k}-\mathbf{q}, \omega)\right]\right\} \\
& +\mathcal{O}\left((t / U)^{3}\right)
\end{aligned}
$$




$$
\begin{aligned}
\mathcal{X}_{0}^{+,+}(\mathbf{q}, \omega) & =\frac{1}{2 \mathcal{N}} \sum_{\mathbf{k}}^{B Z}\left\{\left(\frac{t}{U}\right)^{2} T_{o}(\mathbf{k}) T_{o}(\mathbf{k}-\mathbf{q})\right. \\
& +\left[\mathcal{F}_{1,2}(\mathbf{k}, \mathbf{k}-\mathbf{q}, \omega)+\mathcal{F}_{2,1}(\mathbf{k}, \mathbf{k}-\mathbf{q}, \omega)\right. \\
& \left.\left.-\mathcal{F}_{1,1}(\mathbf{k}, \mathbf{k}-\mathbf{q}, \omega)-\mathcal{F}_{2,2}(\mathbf{k}, \mathbf{k}-\mathbf{q}, \omega)\right]\right\}+\mathcal{O}\left((t / U)^{3}\right)
\end{aligned}
$$

for the matrix elements entering the final expression (53) of the spin-wave dispersion relation. For the remaining matrix elements we obtain instead:

$$
\begin{aligned}
& \mathcal{X}_{0}^{0,0}(\mathbf{q}, \omega)=\frac{1}{4 \mathcal{N}} \sum_{\mathbf{k}}^{B Z}\left\{\left(\frac{t}{U}\right)^{2}\left(T_{o}(\mathbf{k})-T_{o}(\mathbf{k}-\mathbf{q})\right)^{2}\right. \\
& \times\left[\mathcal{F}_{1,2}(\mathbf{k}, \mathbf{k}-\mathbf{q}, \omega)+\mathcal{F}_{2,1}(\mathbf{k}, \mathbf{k}-\mathbf{q}, \omega)\right] \\
& +\left[1-\left(\frac{t}{U}\right)^{2}\left(T_{o}(\mathbf{k})-T_{o}(\mathbf{k}-\mathbf{q})\right)^{2}\right] \\
& \left.\times\left[\mathcal{F}_{1,1}(\mathbf{k}, \mathbf{k}-\mathbf{q}, \omega)+\mathcal{F}_{2,2}(\mathbf{k}, \mathbf{k}-\mathbf{q}, \omega)\right]\right\}+\mathcal{O}\left((t / U)^{3}\right), \\
& \mathcal{X}_{0}^{0,-}(\mathbf{q}, \omega)=\frac{1}{4 \mathcal{N}} \sum_{\mathbf{k}}^{B Z} i \sqrt{2}\left\{\left(\frac{t}{U}\right) \frac{1}{1-\delta}\left(T_{o}(\mathbf{k}-\mathbf{q})-T_{o}(\mathbf{k})\right) \mathcal{F}_{2,1}(\mathbf{k}, \mathbf{k}-\mathbf{q}, \omega)\right. \\
& \left.+\left(\frac{t}{U}\right) \frac{1}{1-\delta}\left[T_{o}(\mathbf{k}) \mathcal{F}_{1,1}(\mathbf{k}, \mathbf{k}-\mathbf{q}, \omega)-T_{o}(\mathbf{k}-\mathbf{q}) \mathcal{F}_{2,2}(\mathbf{k}, \mathbf{k}-\mathbf{q}, \omega)\right]\right\} \\
& +\mathcal{O}\left((t / U)^{3}\right) \\
& \mathcal{X}_{0}^{0, z}(\mathbf{q}, \omega)=\frac{1}{4 \mathcal{N}} \sum_{\mathbf{k}}^{B Z}\left\{\left(\frac{t}{U}\right)^{2}\left(T_{o}^{2}(\mathbf{k})-T_{o}^{2}(\mathbf{k}-\mathbf{q})\right)\right. \\
& \times\left[\mathcal{F}_{2,1}(\mathbf{k}, \mathbf{k}-\mathbf{q}, \omega)-\mathcal{F}_{1,2}(\mathbf{k}, \mathbf{k}-\mathbf{q}, \omega)\right] \\
& +\left[1-\left(\frac{t}{U}\right)^{2}\left(T_{o}^{2}(\mathbf{k})+T_{o}^{2}(\mathbf{k}-\mathbf{q})\right)\right] \\
& \left.\times\left[\mathcal{F}_{1,1}(\mathbf{k}, \mathbf{k}-\mathbf{q}, \omega)-\mathcal{F}_{2,2}(\mathbf{k}, \mathbf{k}-\mathbf{q}, \omega)\right]\right\}+\mathcal{O}\left((t / U)^{3}\right), \\
& \mathcal{X}_{0}^{+, z}(\mathbf{q}, \omega)=\frac{1}{4 \mathcal{N}} \sum_{\mathbf{k}}^{B Z} i \sqrt{2}\left\{\left(\frac{t}{U}\right) \frac{1}{1-\delta}\left(T_{o}(\mathbf{k}-\mathbf{q})+T_{o}(\mathbf{k})\right) \mathcal{F}_{2,1}(\mathbf{k}, \mathbf{k}-\mathbf{q}, \omega)\right. \\
& \left.+\left(\frac{t}{U}\right) \frac{1}{1-\delta}\left[-T_{o}(\mathbf{k}) \mathcal{F}_{1,1}(\mathbf{k}, \mathbf{k}-\mathbf{q}, \omega)-T_{o}(\mathbf{k}-\mathbf{q}) \mathcal{F}_{2,2}(\mathbf{k}, \mathbf{k}-\mathbf{q}, \omega)\right]\right\} \\
& +\mathcal{O}\left((t / U)^{3}\right) \text {, } \\
& \mathcal{X}_{0}^{z, z}(\mathbf{q}, \omega)=\frac{1}{4 \mathcal{N}} \sum_{\mathbf{k}}^{B Z}\left\{\left(\frac{t}{U}\right)^{2}\left(T_{o}(\mathbf{k})+T_{o}(\mathbf{k}-\mathbf{q})\right)^{2}\right. \\
& \times\left[\mathcal{F}_{1,2}(\mathbf{k}, \mathbf{k}-\mathbf{q}, \omega)+\mathcal{F}_{2,1}(\mathbf{k}, \mathbf{k}-\mathbf{q}, \omega)\right]
\end{aligned}
$$




$$
\begin{aligned}
& +\left[1-\left(\frac{t}{U}\right)^{2}\left(T_{o}(\mathbf{k})+T_{o}(\mathbf{k}-\mathbf{q})\right)^{2}\right] \\
& \left.\times\left[\mathcal{F}_{1,1}(\mathbf{k}, \mathbf{k}-\mathbf{q}, \omega)+\mathcal{F}_{2,2}(\mathbf{k}, \mathbf{k k}-\mathbf{q}, \omega)\right]\right\}+\mathcal{O}\left((t / U)^{3}\right) .
\end{aligned}
$$

Note that in the above expressions $\mathcal{F}_{1,1}(\mathbf{k}, \mathbf{k}-\mathbf{q}, \omega)=0$ when the doping parameter $\delta \geq 0$. In addition, $\mathcal{F}_{1,2}$ can be obtained from $\mathcal{F}_{2,1}$ through the symmetry condition (44). Making use of the expansion (35) for the eigenvalues of the mean-field Hamiltonian, we write further

$$
\begin{aligned}
\mathcal{F}_{2,2}(\mathbf{k}, \mathbf{k}-\mathbf{q}) & =\frac{1}{t}\left[f_{F}\left(\epsilon_{2}(\mathbf{k}-\mathbf{q})\right)-f_{F}\left(\epsilon_{2}(\mathbf{k})\right)\right] \frac{1}{T_{e}(\mathbf{k}-\mathbf{q})-T_{e}(\mathbf{k})} \\
& +\mathcal{O}(1 / U)
\end{aligned}
$$

as well as

$$
\begin{aligned}
\mathcal{F}_{2,1}(\mathbf{k}, \mathbf{k}-\mathbf{q}) & =\left[f_{F}\left(\epsilon_{2}(\mathbf{k})\right)-1\right] \frac{1}{U}\left\{\frac{1}{(1-\delta-\tilde{\omega})}\right. \\
& -\left(\frac{t}{U}\right) \frac{1}{(1-\delta-\tilde{\omega})^{2}}\left[T_{e}(\mathbf{k})-T_{e}(\mathbf{k}-\mathbf{q})\right]+\left(\frac{t}{U}\right)^{2}\left[\frac{1}{(1-\delta-\tilde{\omega})^{2}}\right. \\
& \times\left(-T_{o}^{2}(\mathbf{k})-T_{o}^{2}(\mathbf{k}-\mathbf{q})-2 m_{2}^{(2)}\right) \\
& \left.\left.+\frac{1}{(1-\delta-\tilde{\omega})^{3}}\left(T_{e}(\mathbf{k})-T_{e}(\mathbf{k}-\mathbf{q})\right)^{2}\right]\right\} \\
& +\mathcal{O}\left(t^{3} / U^{4}\right)
\end{aligned}
$$

with $\tilde{\omega}=\omega / U$, where the expansion (B2) of the Fermi function has still to be inserted.

\section{APPENDIX C: SUMS OVER THE TWO-DIMENSIONAL BZ IN THE SMALL $\delta$ LIMIT}

In this Appendix, we develop a method suitable for performing the $\mathbf{k}$ summation over the relevant portions of the Brillouin zone in powers of the doping parameter $\delta$. This is, in turn, justified by the fact that $\delta$ in the spiral phase is of order $t / U$, as shown in Appendix B.

The typical integral to be evaluated is of the form:

$$
\mathcal{I}(\gamma)=\frac{1}{\mathcal{N}} \sum_{\mathbf{k}}^{B Z} g(\mathbf{k}) \Theta\left(T_{e}(\mathbf{k})+\gamma\right)
$$

where $T_{e}(\mathbf{k})=2 \cos (Q / 2)\left(\cos k_{x}+\cos k_{y}\right)$ for the diagonal spiral solution we are considering and $\gamma$ is a parameter (which depends on the chemical potential $\mu^{(1)}$ at the order $\mathcal{O}\left[(t / U)^{0}\right]$ ) that in the following we shall simply call $\mu$ for simplicity. Introducing the notation $f_{\mu}$ such that $\gamma=4 \cos (Q / 2)\left(1-f_{\mu} / 4\right)$ for any given $Q$ value, recalling the definition of the $\Theta$ function, and considering that $\cos (Q / 2)<0$ in our solution, we set further:

$$
\mathcal{I}(\gamma)=\frac{1}{\mathcal{N}} \sum_{\cos k_{x}+\cos k_{y} \leq 2-f_{\mu} / 2} g(\mathbf{k}) .
$$

Quite generally, we can introduce a polar representation for the two-dimensional wave vector $\mathbf{k}$ and determine for each value of the polar angle $\phi$ the magnitude $k(\phi)$, such that the equality

$$
\cos (k(\phi) \cos \phi)+\cos (k(\phi) \sin \phi)=2-\frac{f_{\mu}}{2}
$$

is satisfied. In this way we rewrite the integral (C2) as follows:

$$
\mathcal{I}(\gamma)=\frac{1}{4 \pi^{2}} \int_{B Z} d k_{x} d k_{y} g\left(k_{x}, k_{y}\right)-\frac{1}{4 \pi^{2}} \int_{0}^{2 \pi} d \phi \int_{0}^{k(\phi)} d k k g(k, \phi)
$$


with a slight (albeit harmless) abuse of notation for the function $g$.

In practice, when $f_{\mu}$ is small compared to unity (as it is the case for small $\delta$ ) it is convenient to determine $k(\phi)$ in powers of $f_{\mu}$ by expanding $\cos (k(\phi) \cos \phi)+\cos (k(\phi) \sin \phi)$ in a Taylor series about $k=0$. For instance, at the lowest order we obtain from Eq. (C3):

$$
k(\phi)^{2}+\mathcal{O}\left(k(\phi)^{4}\right)=f_{\mu}
$$

which gives $k(\phi)^{2}=f_{\mu}+\mathcal{O}\left(f_{\mu}^{2}\right)$. At the next significant order we obtain instead:

$$
k(\phi)^{2}-\frac{\left(\sin ^{4} \phi+\cos ^{4} \phi\right)}{12} k(\phi)^{4}+\mathcal{O}\left(k(\phi)^{6}\right)=f_{\mu}
$$

which gives

$$
k(\phi)^{2}=f_{\mu}+\frac{\left(\sin ^{4} \phi+\cos ^{4} \phi\right)}{12} f_{\mu}^{2}+\mathcal{O}\left(f_{\mu}^{3}\right) .
$$

Consider, for instance, the first of Eqs. (B7), which is of the form (C1) with $g(\mathbf{k})=1$ and $\gamma=m_{2}^{(1)}-\mu^{(1)}$. Equation (C4) now becomes:

$$
\delta=\frac{1}{8 \pi^{2}} \int_{0}^{2 \pi} d \phi k(\phi)^{2}
$$

Introducing the notation $f_{\mu}$ as above, for small values of $\delta(>0)$ we obtain:

$$
\delta=\frac{1}{4 \pi}\left[f_{\mu}+\frac{1}{16} f_{\mu}^{2}+\mathcal{O}\left(f_{\mu}^{3}\right)\right]
$$

where use has been made of Eq. (C7). Inverting this relation we obtain eventually:

$$
f_{\mu}=4 \pi \delta-\pi^{2} \delta^{2}+\mathcal{O}\left(\delta^{3}\right),
$$

which confirms the fact that $f_{\mu}=\mathcal{O}(\delta)$.

In general, the case of a $\mathbf{k}$-dependent $g$ can be treated by expanding $g(\mathbf{k})$ about $|\mathbf{k}|=0$, since the last integral on the rignt-hand side of Eq. ( $\mathrm{C} 4)$ is restricted to $|\mathbf{k}|<k(\phi)=\mathcal{O}\left(\delta^{1 / 2}\right)$, and by retaining the relevant orders in $\delta$ in the final expression. The integral over the whole BZ (i.e., the first term on the rignt-hand side of Eq. (C4)), on the other hand, can usually be performed analytically.

The Brillouin zone sums involving the Dirac delta functions or its derivatives can be further evaluated according to the following device. Writing

$$
\begin{aligned}
\delta\left(T_{e}(\mathbf{k})+\gamma\right) & =\frac{\partial}{\partial \gamma} \Theta\left(T_{e}(\mathbf{k})+\gamma\right) \\
& =\frac{1}{\cos (Q / 2)} \frac{\partial}{\partial f_{\mu}} \Theta\left(T_{e}(\mathbf{k})+\gamma\right),
\end{aligned}
$$

we obtain for the Brillouin zone sum

$$
\begin{aligned}
& \frac{1}{\mathcal{N}} \sum_{\mathbf{k}}^{B Z} g(\mathbf{k}) \delta^{(n)}\left(T_{e}(\mathbf{k})+m_{2}^{(1)}-\mu^{(1)}\right) \\
& =\frac{1}{(\cos (Q / 2))^{(n+1)}} \frac{\partial^{(n+1)}}{\partial\left(f_{\mu}^{(1)}\right)^{(n+1)}} \frac{1}{\mathcal{N}} \sum_{\mathbf{k}}^{B Z} g(\mathbf{k}) \Theta\left(T_{e}(\mathbf{k})+m_{2}^{(1)}-\mu^{(1)}\right) \\
& =-\frac{1}{(\cos (Q / 2))^{(n+1)}} \frac{\partial^{(n+1)}}{\partial\left(f_{\mu}^{(1)}\right)^{(n+1)}} \frac{1}{4 \pi^{2}} \int_{0}^{2 \pi} d \phi \int_{O}^{k(\phi)} d k k g(k, \phi)
\end{aligned}
$$

since the first term on the right-hand side of Eq. (C4) does not depend on $f_{\mu}$.

As an example, we evaluate the expression 


$$
\frac{1}{\mathcal{N}} \sum_{\mathbf{k}}^{B Z} T_{o}^{2}(\mathbf{k}) \delta\left(T_{e}(\mathbf{k})+m_{2}^{(1)}-\mu^{(1)}\right)
$$

which appears in the first of Eqs. (B8). According to Eq. (C11), we first evaluate the expression (cf. also Eq. (C4)):

$$
\begin{aligned}
& \frac{1}{\mathcal{N}} \sum_{\mathbf{k}}^{B Z} T_{o}^{2}(\mathbf{k}) \Theta\left(T_{e}(\mathbf{k})+m_{2}^{(1)}-\mu^{(1)}\right) \\
& =4 \sin ^{2}(Q / 2)-\frac{1}{\pi^{2}} \sin ^{2}(Q / 2) \int_{0}^{2 \pi} d \phi \int_{0}^{k(\phi)} d k k(\sin (k \cos \phi)+\sin (k \sin \phi))^{2} .
\end{aligned}
$$

Expanding the factor within parentheses in the last integral in powers of $k$, as explained below Eq. (C10), we obtain:

$$
\begin{aligned}
& \frac{1}{\mathcal{N}} \sum_{\mathbf{k}}^{B Z} T_{o}^{2}(\mathbf{k}) \Theta\left(T_{e}(\mathbf{k})+m_{2}^{(1)}-\mu^{(1)}\right) \\
& =4 \sin ^{2}(Q / 2)-\frac{1}{\pi^{2}} \sin ^{2}(Q / 2) \int_{0}^{2 \pi} d \phi \int_{0}^{k(\phi)} d k k\left(k^{2}(\cos \phi+\sin \phi)^{2}+\mathcal{O}\left(k^{4}\right)\right) \\
& =\sin ^{2}(Q / 2)\left(4-8 \pi \delta^{2}+\mathcal{O}\left(\delta^{3}\right)\right) .
\end{aligned}
$$

Using Eq. (C11) we obtain eventually:

$$
\begin{aligned}
& \frac{1}{\mathcal{N}} \sum_{\mathbf{k}}^{B Z} T_{o}^{2}(\mathbf{k}) \delta\left(T_{e}(\mathbf{k})+m_{2}^{(1)}-\mu^{(1)}\right) \\
& =\frac{1}{\cos (Q / 2)} \frac{\partial}{\partial f_{\mu}} \frac{1}{\mathcal{N}} \sum_{\mathbf{k}}^{B Z} T_{o}^{2}(\mathbf{k}) \Theta\left(T_{e}(\mathbf{k})+m_{2}^{(1)}-\mu^{(1)}\right) \\
& =-\frac{\sin ^{2}(Q / 2)}{\cos (Q / 2)} \frac{1}{\pi} f_{\mu}+\mathcal{O}\left(\left(f_{\mu}^{2}\right)=-4 \delta \frac{\sin ^{2}(Q / 2)}{\cos (Q / 2)}+\mathcal{O}\left(\delta^{2}\right) .\right.
\end{aligned}
$$

With these prescriptions, the results (B10), (B11), and (B12) are readily obtained.

It was shown in Section 3 that, as far as the matrix elements of the correlation function are concerned, only the explicit expressions of $d(\mathbf{q}, \omega)$ and $e(\mathbf{q}, \omega)$ are required at the order in $t / U$ we are considering in this paper. Writing

$$
\begin{aligned}
d(\mathbf{q}, \omega) & =-1-\tilde{\omega}+\alpha(\mathbf{q})+\mathcal{O}\left((t / U)^{3}\right) \\
& =I(\mathbf{q}, \omega)-J(\mathbf{q}, \omega)-K(\mathbf{q}, \omega)+M(\mathbf{q}, \omega)
\end{aligned}
$$

and

$$
e(\mathbf{q}, \omega)=L(\mathbf{q}, \omega)+L(-\mathbf{q},-\omega)+N(\mathbf{q}, \omega)
$$

where the quantities $I, J, \cdots$ are specified below, we obtain for the sums over the wave vector $\mathbf{k}$ using the method described in this Appendix:

$$
\begin{aligned}
I(\mathbf{q}, \omega) & \equiv \frac{U}{\mathcal{N}} \sum_{\mathbf{k}}^{B Z} \mathcal{F}_{21}(\mathbf{k}, \mathbf{k}-\mathbf{q}, \omega)=\frac{-1+\delta}{1-\tilde{\omega}-\delta} \\
& +\left(\frac{t}{U}\right) 2 \delta \cos (Q / 2)\left(\cos q_{x}+\cos q_{y}\right) \\
& +\left(\frac{t}{U}\right)^{2} 4 \cos ^{2}(Q / 2)\left(\cos q_{x}+\cos q_{y}\right)+\mathcal{O}\left((t / U)^{3}\right) \\
J(\mathbf{q}, \omega) & \equiv \frac{U}{\mathcal{N}}\left(\frac{t}{U}\right)^{2} \sum_{\mathbf{k}}^{B Z} T_{o}^{2}(\mathbf{k}) \mathcal{F}_{21}(\mathbf{k}, \mathbf{k}-\mathbf{q}, \omega) \\
& =-4\left(\frac{t}{U}\right)^{2} \sin ^{2}(Q / 2)+\mathcal{O}\left((t / U)^{3}\right)
\end{aligned}
$$




$$
\begin{aligned}
& K(\mathbf{q}, \omega) \equiv \frac{U}{\mathcal{N}}\left(\frac{t}{U}\right)^{2} \sum_{\mathbf{k}}^{B Z} T_{o}^{2}(\mathbf{k}-\mathbf{q}) \mathcal{F}_{21}(\mathbf{k}, \mathbf{k}-\mathbf{q}, \omega) \\
& =-4\left(\frac{t}{U}\right)^{2} \sin ^{2}(Q / 2)+\mathcal{O}\left((t / U)^{3}\right), \\
& L(\mathbf{q}, \omega) \equiv \frac{U}{\mathcal{N}}\left(\frac{t}{U}\right)^{2} \sum_{\mathbf{k}}^{B Z} T_{o}(\mathbf{k}) T_{o}(\mathbf{k}-\mathbf{q}) \mathcal{F}_{21}(\mathbf{k}, \mathbf{k}-\mathbf{q}, \omega) \\
& =-2\left(\frac{t}{U}\right)^{2} \sin ^{2}(Q / 2)\left(\cos q_{x}+\cos q_{y}\right)+\mathcal{O}\left((t / U)^{3}\right), \\
& M(\mathbf{q}, \omega) \equiv \frac{U}{\mathcal{N}}\left(\frac{t}{U}\right)^{2} \sum_{\mathbf{k}}^{B Z} T_{o}^{2}(\mathbf{k}-\mathbf{q}) \mathcal{F}_{22}(\mathbf{k}, \mathbf{k}-\mathbf{q}, \omega) \\
& =-2 \delta\left(\frac{t}{U}\right) \frac{\sin ^{2}(Q / 2)\left(\sin q_{x}+\sin q_{y}\right)^{2}}{\cos (Q / 2)\left(\cos q_{x}+\cos q_{y}-2\right)}+\mathcal{O}\left((t / U)^{3}\right), \\
& N(\mathbf{q}, \omega) \equiv \frac{U}{\mathcal{N}}\left(\frac{t}{U}\right)^{2} \sum_{\mathbf{k}}^{B Z} T_{o}(\mathbf{k}) T_{o}(\mathbf{k}-\mathbf{q}) \mathcal{F}_{22}(\mathbf{k}, \mathbf{k}-\mathbf{q}, \omega) \\
& =\left(\frac{t}{U}\right)^{2} \mathcal{O}(\delta)=\mathcal{O}\left((t / U)^{3}\right) \text {, }
\end{aligned}
$$

where these results hold for $|\mathbf{q}| \gg k_{F}$, as discussed in Section 3-B.

For the relevant matrix elements (C16) and (C17) we obtain eventually :

$$
\begin{aligned}
d(\mathbf{q}, \omega) & =-1-\tilde{\omega}+\left(\frac{t}{U}\right)^{2} 4 \cos ^{2}(Q / 2)\left(\cos q_{x}+\cos q_{y}-2\right)+8\left(\frac{t}{U}\right)^{2} \sin ^{2}(Q / 2) \\
& +2\left(\frac{t}{U}\right) \delta \cos (Q / 2)\left(\cos q_{x}+\cos q_{y}-2\right) \\
& -2\left(\frac{t}{U}\right) \delta \frac{\sin ^{2}(Q / 2)\left(\sin q_{x}+\sin q_{y}\right)^{2}}{\cos (Q / 2)\left(\cos q_{x}+\cos q_{y}-2\right)}+\mathcal{O}\left((t / U)^{3}\right)
\end{aligned}
$$

and

$$
e(\mathbf{q}, \omega)=-4\left(\frac{t}{U}\right)^{2} \sin ^{2}(Q / 2)\left(\cos q_{x}+\cos q_{y}\right)+\mathcal{O}\left((t / U)^{3}\right)
$$

\section{APPENDIX D: HEISENBERG SPIN WAVES FOR A SPIRAL CONFIGURATION}

In this Appendix, we give a simplified derivation of the spin-wave dispersion relation for a two-dimensional Heisenberg antiferromagnet in the presence of a spiral incommensurate magnetic ground state. Although the form of this spectrum is well known, 10.21 it is worthed to give here a compact derivation in terms of the set of local spin quantization axes utilized in Section 3 and in Appendix A.

We consider the Heisenberg Hamiltonian

$$
H=\frac{1}{2} \sum_{i, j} J_{i, j} \mathbf{S}_{i} \cdot \mathbf{S}_{j}
$$

for spin $1 / 2$, where the sum over the lattice sites $i$ and $j$ extends in principle over distant neighbors. We transform next the spin locally as follows: 


$$
S_{i}^{\alpha} \rightarrow \sum_{\beta} T_{\alpha \beta}^{(i)} S_{i}^{\beta}
$$

where $T^{(i)}$ represents the $3 \times 3$ matrix given by the spin part of $(\mathrm{A12})$. The Hamiltonian (D1) then becomes:

$$
H=\frac{1}{2} \sum_{i, j} J_{i, j} \sum_{\alpha, \beta} S_{i}^{\alpha} T_{\alpha \beta}^{(j i)} S_{j}^{\beta}
$$

where $T^{(j i)}$ contains the difference $\theta_{j}-\theta_{i}$ in the place of $\theta_{i}$ of Eq. (A12).

Writing further

$$
S_{i}^{x}=\frac{1}{2}\left(S_{i}^{+}+S_{i}^{-}\right), S_{i}^{y}=\frac{1}{2 i}\left(S_{i}^{+}-S_{i}^{-}\right),
$$

and carrying out the standard Holstein-Primakoff transformation

$$
\begin{aligned}
S_{i}^{ \pm} & \cong \sqrt{2 S} a_{i}^{ \pm} \\
S_{i}^{z} & =a_{i}^{\dagger} a_{i}-S=n_{i}-S
\end{aligned}
$$

at the leading order away from perfect alignment (and for large values of $S$ ), the Hamiltonian (D3) becomes:

$$
\begin{aligned}
H & \cong \frac{S^{2}}{2} \sum_{i, j} J_{i, j} \cos \left(\theta_{j}-\theta_{i}\right)-S \sum_{i, j} n_{i} J_{i, j} \cos \left(\theta_{j}-\theta_{i}\right) \\
& +\frac{S}{4} \sum_{i, j} J_{i, j}\left\{\left(a_{i}^{\dagger} a_{j}+a_{j}^{\dagger} a_{i}\right)\left[\cos \left(\theta_{j}-\theta_{i}\right)+1\right]\right. \\
& \left.+\left(a_{i}^{\dagger} a_{j}^{\dagger}+a_{i} a_{j}\right)\left[\cos \left(\theta_{j}-\theta_{i}\right)-1\right]\right\} .
\end{aligned}
$$

We introduce at this point the lattice Fourier transform, specify the choice $\theta_{i}=\mathbf{Q} \cdot \mathbf{R}_{i}$, and write:

$$
\sum_{i, j} a_{i} b_{j} \cos \mathbf{Q} \cdot\left(\mathbf{R}_{j}-\mathbf{R}_{i}\right) J_{i, j}=\frac{1}{2} \sum_{\mathbf{k}}^{B Z} a(\mathbf{k}) b(-\mathbf{k})(J(\mathbf{k}-\mathbf{Q})+J(\mathbf{k}+\mathbf{Q})),
$$

where $a_{i}$ and $b_{j}$ is an arbitrary pair of operators and where

$$
J(\mathbf{k})=\sum_{\mathbf{R}} \exp (-i \mathbf{k} \cdot \mathbf{R}) J(\mathbf{R}) .
$$

With the notation

$$
\alpha(\mathbf{k}) \equiv-S J(\mathbf{Q})+\frac{S}{2}\left(J(\mathbf{k})+\frac{J(\mathbf{k}+\mathbf{Q})+J(\mathbf{k}-\mathbf{Q})}{2}\right)
$$

and

$$
\beta(\mathbf{k}) \equiv-\frac{S}{2}\left(J(\mathbf{k})-\frac{J(\mathbf{k}+\mathbf{Q})+J(\mathbf{k}-\mathbf{Q})}{2}\right)
$$

for any given value of $\mathbf{Q}$, the Hamiltonian (D6) reduces to the form:

$$
\begin{aligned}
H & \cong \frac{\mathcal{N} S^{2}}{2} J(\mathbf{Q})+\sum_{\mathbf{k}}^{B Z}\left[\alpha(\mathbf{k}) a^{\dagger}(\mathbf{k}) a(\mathbf{k})\right. \\
& \left.+\frac{\beta(\mathbf{k})}{2}\left(a^{\dagger}(\mathbf{k}) a^{\dagger}(-\mathbf{k})+a(\mathbf{k}) a(-\mathbf{k})\right)\right] .
\end{aligned}
$$

The standard Bogoliubov transformation can be used at this point to diagonalize the Hamiltonian (D11). Writing

$$
a(\mathbf{k})=u(\mathbf{k}) b(\mathbf{k})+v(\mathbf{k}) b^{\dagger}(-\mathbf{k})
$$


where $u(\mathbf{k})=\cosh \psi(\mathbf{k})$ and $v(\mathbf{k})=\sinh \psi(\mathbf{k})$ with

$$
\tanh 2 \psi(\mathbf{k})=-\frac{\beta(\mathbf{k})}{\alpha(\mathbf{k})} \equiv \gamma(\mathbf{k}),
$$

the Hamiltonian (D11) becomes eventually:

$$
H=E_{0}+E_{1}+\sum_{\mathbf{k}}^{B Z} \varepsilon(\mathbf{k}) b^{\dagger}(\mathbf{k}) b(\mathbf{k}) .
$$

In this expression:

$$
\begin{gathered}
E_{0}=\frac{\mathcal{N} S^{2}}{2} J(\mathbf{Q}) \\
E_{1}=-\sum_{\mathbf{k}}\left(\alpha(\mathbf{k}) v^{2}(\mathbf{k})+u(\mathbf{k}) v(\mathbf{k}) \beta(\mathbf{k})\right)
\end{gathered}
$$

and

$$
\begin{aligned}
\varepsilon(\mathbf{k}) & =\sqrt{\alpha^{2}(\mathbf{k})-\beta^{2}(\mathbf{k})} \\
& =S \sqrt{(J(\mathbf{Q})-J(\mathbf{k}))\left(J(\mathbf{Q})-\frac{J(\mathbf{k}+\mathbf{Q})+J(\mathbf{k}-\mathbf{Q})}{2}\right)} .
\end{aligned}
$$

The spectrum (D17) coincides (apart for a different normalization of $J$ ) with that given in Ref.21 (cf. in particular Eq. (67) therein). Note from Eq. (D17) the presence of a Goldstone mode for $\mathbf{k}=0$ and $\mathbf{k}= \pm \mathbf{Q}$ (owing to the symmetry $J(-\mathbf{k})=J(\mathbf{k}))$.

In the classical limit (that is, for large values of $S$ ), minimization of the ground-state energy is equivalent to finding the minimum of $J(\mathbf{Q})$. In this way, the appropriate value of $\mathbf{Q}$ is determined. For instance, when second $\left(J_{2}\right)$ and third $\left(J_{3}\right)$ nearest-neighbor couplings are included besides the nearest-neighbor antiferromagnetic coupling $\left(J_{1}\right), J(\mathbf{Q})$ reads:

$$
J(\mathbf{Q})=J_{1}\left(\cos Q_{x}+\cos Q_{y}\right)+2 J_{2} \cos Q_{x} \cos Q_{y}+J_{3}\left(\cos 2 Q_{x}+\cos 2 Q_{y}\right)
$$

(with the wave vectors measured in units of the inverse of the lattice spacing). In this case, the diagonal spiral configuration is stable for $J_{3}>J_{1} / 4-J_{2} / 2$ and $J_{3}>J_{2} / 2$, implying that $J_{3}$ must be nonvanishing for the spiral phase to be stable.22 The corresponding spin-wave spectrum (D17) for $J_{3}=J_{2}=J_{1} / 5$ and $S=1 / 2$ is shown in Fig. 4 (full line), where the antiferromagnetic spectrum with $\mathbf{Q}=(\pi, \pi)$ and $J_{3}=J_{2}=0$ (broken line) is also shown for comparison.

\section{APPENDIX E: RKKY SPIN WAVES FOR A SPIRAL CONFIGURATION}

In this Appendix, we show how the part of the dispersion relation (57) of the text containing the trigonometric functions in the denominator originates from the Rudermap-Kittel-Kasuya-Yosida (RKKY) interaction between two localized spins and mediated by the conduction electrons.23 11 The long-range nature of this interaction is readily evidenced by expanding formally the denominator in Eq. (57) as a a power series in $\cos q_{x}$ and $\cos q_{y}$, so that an infinite number of Heisenberg-like terms appears.

We follow here the treatment by Mattis 23 and consider the interaction Hamiltonian between the localized spin operators $\mathbf{S}_{i}$ (associated with the valence (filled) band) and the itinerant spin operators $\mathbf{s}_{c}\left(\mathbf{R}_{i}\right.$ ) (associated with the conduction band), where the suffix $i$ specifies the lattice site. We write accordingly:

$$
H_{\mathrm{exc}}=-I \sum_{i} \mathbf{S}_{i} \cdot \mathbf{s}_{c}\left(\mathbf{R}_{i}\right)
$$

where $I$ is an exchange integral.

For a generic spin- $1 / 2$ operator at site $i$ associated with the conduction electrons we write: 


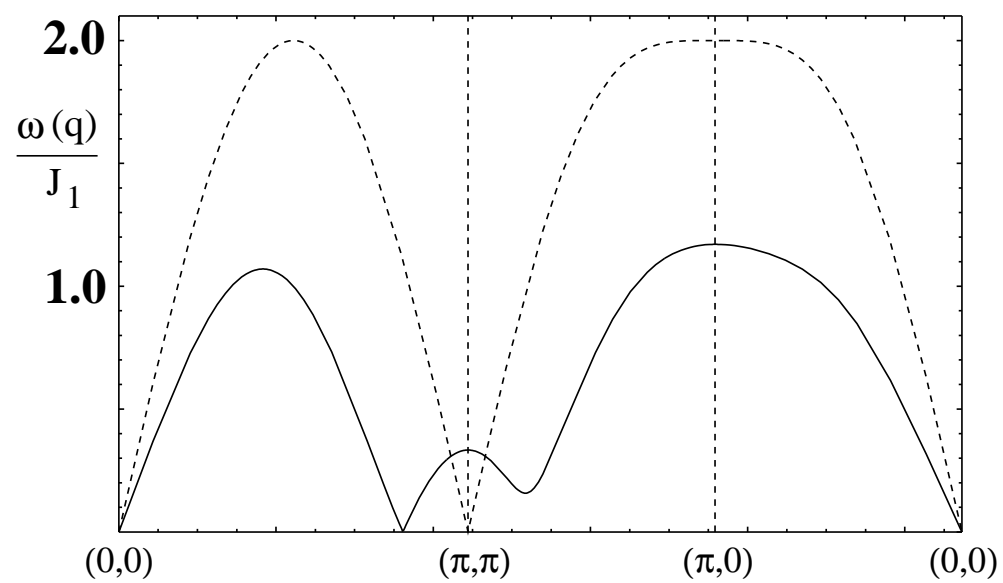

FIG. 4. Spin-wave spectrum for the Heisenberg Hamiltonian with a diagonal-spiral spin configuration and couplings up to third nearest neighbors specified in the text (full line). The antiferromagnetic spectrum with nearest-neighbor coupling only is also shown for comparison (broken line).

$$
s_{i}^{\mu}=\frac{1}{2 \mathcal{N}} \sum_{\mathbf{k}, \mathbf{q}} \sum_{\xi, \xi^{\prime}} \mathrm{e}^{-i \mathbf{q} \cdot \mathbf{R}_{i}} d_{\mathbf{k}+\mathbf{q} \xi}^{\dagger} \sigma_{\xi, \xi^{\prime}}^{\mu} d_{\mathbf{k} \xi^{\prime}}
$$

where $\mu=(+,-, z)$ and $d_{i \xi}$ are the destruction operators along the local spin-quantization axes. Combining these operators with the eigenvectors of the mean-field Hubbard Hamiltonian [cf. Eq. (A8)] and recalling the notation (A15), we cast Eq. (E2) in the form:

$$
s_{i}^{\mu}=\frac{1}{2 \mathcal{N}} \sum_{\mathbf{k}, \mathbf{q}} \sum_{r, r^{\prime}} \mathrm{e}^{-i \mathbf{q} \cdot \mathbf{R}_{i}} \gamma_{\mathbf{k}+\mathbf{q}, r}^{\dagger} F_{r, r^{\prime}}^{\mu}(\mathbf{k}+\mathbf{q}, \mathbf{k}) \gamma_{\mathbf{k}, r^{\prime}} .
$$

The restriction to the conduction band implies that $r=r^{\prime}=2$ in Eq. (E3) when $\delta>0$. In this case the Hamiltonian (E1) becomes:

$$
H_{\mathrm{exc}}=-\frac{I}{2 \mathcal{N}} \sum_{i} \sum_{\mu}\left(S_{i}^{\mu}\right)^{\dagger}\left(\sum_{\mathbf{k}, \mathbf{q}} e^{-i \mathbf{q} \cdot \mathbf{R}_{i}} \gamma_{\mathbf{k}+\mathbf{q}, 2}^{\dagger} F_{22}^{\mu}(\mathbf{k}+\mathbf{q}, \mathbf{k}) \gamma_{\mathbf{k}, 2}\right)
$$

Applying at this point the standard procedure 23 to obtain the energy shift of second-order in the Hamiltonian (E4), the following RKKY-type effective Hamiltonian results:

$$
H^{R K K Y}=\sum_{i j} \sum_{\mu \nu}\left(S_{i}^{\mu}\right)^{\dagger} J_{i j}^{\mu \nu} S_{j}^{\nu}
$$

with the notation

$$
J_{i j}^{\mu \nu}=\frac{I^{2}}{4 \mathcal{N}^{2}} \sum_{k<k_{F}} \sum_{|\mathbf{k}+\mathbf{q}|>k_{F}} \mathrm{e}^{-i \mathbf{q} \cdot\left(\mathbf{R}_{i}-\mathbf{R}_{j}\right)} \frac{F_{22}^{\mu}(\mathbf{k}+\mathbf{q}, \mathbf{k}) F_{22}^{\nu *}(\mathbf{k}+\mathbf{q}, \mathbf{k})}{\epsilon_{2}(\mathbf{k})-\epsilon_{2}(\mathbf{k}+\mathbf{q})}
$$

Note that the expression (E6) does not vanish even when sites $i$ and $j$ are far apart. Note also that the energy denominator on the right-hand side never vanish by construction.

There remains to obtain the spin-wave dispersion associated with the spin Hamiltonian (E5). To this end, we introduce in Eq. (E5) the usual Holstein-Primakoff transformation for the spin operators and truncate the expansion in the bosonic operators $a$ and $a^{\dagger}$ to quadratic order [cf. Eq. (D5)], to obtain:

$$
\begin{aligned}
H^{R K K Y} & =E_{0}+S \sum_{|\mathbf{q}| \gg k_{F}}\left[\left(J^{++}(\mathbf{q})+J^{--}(-\mathbf{q})\right) a^{\dagger}(\mathbf{q}) a(\mathbf{q})\right. \\
& \left.+J^{+-}(\mathbf{q}) a^{\dagger}(\mathbf{q}) a^{\dagger}(-\mathbf{q})+J^{-+}(-\mathbf{q}) a(\mathbf{q}) a(-\mathbf{q})\right]
\end{aligned}
$$


where now

$$
E_{0}=S^{2} \sum_{|\mathbf{q}| \gg k_{F}} J^{z z}(\mathbf{q})+S \sum_{|\mathbf{q}| \gg k_{F}} J^{--}(\mathbf{q})
$$

with the restriction $|\mathbf{q}| \gg k_{F}$ replacing the weaker condition $|\mathbf{k}+\mathbf{q}|>k_{F}$ (cf. Section 3 -B). Note that in Eq. (E7) we have introduced the notation

$$
J^{\mu \nu}(\mathbf{q})=\frac{I^{2}}{4 \mathcal{N}} \sum_{|\mathbf{k}|<k_{F}} \frac{F_{22}^{\mu}(\mathbf{k}+\mathbf{q}, \mathbf{k}) F_{22}^{\nu *}(\mathbf{k}+\mathbf{q}, \mathbf{k})}{\epsilon_{2}(\mathbf{k})-\epsilon_{2}(\mathbf{k}+\mathbf{q})}
$$

and used the identity $\sum_{i} J_{i j}^{\mu \nu}=0$ for any given $j$.

The quadratic Hamiltonian (E7) is then diagonalized by the standard Bogoliubov transformation (cf. Appendix D). The resulting spin-wave spectrum has the form:

$$
\omega^{R K K Y}(\mathbf{q})=S\left[\left(J^{++}(\mathbf{q})+J^{--}(-\mathbf{q})\right)^{2}-4 J^{+-}(\mathbf{q}) J^{-+}(-\mathbf{q})\right]^{1 / 2} .
$$

To proceed further, we need to specify the quantities $F_{22}^{+}, F_{22}^{-}$, and $\epsilon_{2}$ entering the definition (E9), at the relevant order in the small parameter $t / U$. Using Eqs. (35)-(38) we obtain for $\delta>0$ :

$$
\begin{aligned}
& F_{22}^{+}(\mathbf{k}+\mathbf{q}, \mathbf{k})=i \sqrt{2}\left(\frac{t}{U}\right) T_{o}(\mathbf{k}+\mathbf{q})+\mathcal{O}\left((t / U)^{2}\right) \\
& F_{22}^{-}(\mathbf{k}+\mathbf{q}, \mathbf{k})=-i \sqrt{2}\left(\frac{t}{U}\right) T_{o}(\mathbf{k})+\mathcal{O}\left((t / U)^{2}\right)
\end{aligned}
$$

as well as

$$
\epsilon_{2}(\mathbf{k})-\epsilon_{2}(\mathbf{k}+\mathbf{q})=t\left(T_{e}(\mathbf{k})-T_{o}(\mathbf{k}+\mathbf{q})\right)+\mathcal{O}\left((t / U)^{2}\right)
$$

where we have approximated $2 m_{2}^{(0)}=1-\delta \cong 1$.

The method developed in Appendix $\mathrm{C}$ can be used at this point to perform the sum over $\mathbf{k}$ in Eq. (E9). The result is:

$$
\begin{gathered}
J^{++}(\mathbf{q}) \cong-I^{2} \frac{t}{U^{2}} \delta \frac{\sin ^{2}(Q / 2)}{\cos (Q / 2)} \frac{\left(\sin q_{x}+\sin q_{y}\right)^{2}}{\cos q_{x}+\cos q_{y}-2}, \\
J^{--}(\mathbf{q}) \cong-I^{2} \frac{t}{U^{2}} \mathcal{O}\left(\delta^{2}\right), \\
J^{+-}(\mathbf{q})=J^{-+}(\mathbf{q}) \cong I^{2} \frac{t}{U^{2}} \mathcal{O}\left(\delta^{2}\right) .
\end{gathered}
$$

At the lowest order, only $J^{++}$contributes to Eq. (E10), which then reduces to:

$$
\omega^{R K K Y}(\mathbf{q}) \cong S I^{2} \frac{t}{U^{2}} \delta \frac{\sin ^{2}(Q / 2)\left(\sin q_{x}+\sin q_{y}\right)^{2}}{|\cos (Q / 2)|\left(2-\cos q_{x}-\cos q_{y}\right)} .
$$

The self-consistency equation (43) relating $Q$ to $\delta$ and $t / U$ for the diagonal spiral configuration can eventually be used, to yield (for $S=1 / 2$ ):

$$
\omega^{R K K Y}(\mathbf{q}) \cong J_{\text {eff }} \frac{\left(\sin q_{x}+\sin q_{y}\right)^{2}}{2-\cos q_{x}-\cos q_{y}}
$$

with $J_{\text {eff }}$ given by Eq. (58) of the text and where we have set $I=2 U$. Note that Eq. (E17) coincides with Eq. (60) of the text, which was obtained by setting "by hand" $\mathcal{F}_{21}=\mathcal{F}_{12}=0$ in the full calculation. Note also that the expression (E17) involves transverse spin components only, akin the general expression (53) of the text. 
${ }^{1}$ Cf. the review article by M.A. Kastner, R.J. Birgeneau, S. Shirane, and Y. Endoh, Rev. Mod. Phys. 70, 897 (1998), and references quoted therein.

2 T.R. Thurston et al., Phys. Rev. B 40, 4585 (1989).

${ }^{3}$ S. M. Hayden, G. Aeppli, H. A. Mook, T. G. Perring, T. E. Mason, S-W. Cheong, and Z. Fisk, Phys. Rev. Lett. 76, 1344 (1996).

${ }^{4}$ H.A. Mook, Phys. Rev. Lett. 46, 508 (1981), and references quoted therein.

${ }^{5}$ C. Zhou and H.J. Schulz, Phys. Rev. B 52, R11557 (1995).

${ }^{6}$ B.L. Shraiman and E.D. Siggia, Phys. Rev. Lett. 62, 1564 (1989); H. J. Schulz, Phys. Rev. Lett. 64, 1445 (1990).

${ }^{7}$ W. Brenig, Ann. Physik 5, 123 (1996).

8 Jun-ichi Igarashi and P. Fulde, Phys. Rev. B 45, 10419 (1992).

${ }^{9}$ J. M. Tranquada, B. J. Sternlieb, J. D. Axe, Y. Nakamura, and S. Uchida, Nature 375, 561 (1995). For an analysis of photoemission experiments in the striped phase see also M. G. Zacher, R. Eder, E. Arrigoni, and W. Hanke, preprint (cond-mat/0005473).

10 F. Keffer, Handbuch der Physik, Vol. 28, 1 (1966).

${ }^{11}$ M.A. Ruderman and C. Kittel, Phys. Rev. 96, 99 (1954). For an extension to the two-dimensional case, see M.T. Béal-Monod, Phys. Rev. B 36, 8835 (1987).

12 A. Muramatsu and R. Zeyher, Nucl. Phys. B 346, 387 (1990).

${ }^{13}$ W. Heisenberg, Z. Phys, 49, 619 (1928).

14 E. Arrigoni and G.C. Strinati, Phys. Rev. B 45, 7816 (1992).

${ }^{15}$ G. Baym and L.P. Kadanoff, Phys. Rev. 124, 287 (1961); G. Baym, Phys. Rev. 127, 1391 (1962).

${ }^{16}$ E. Arrigoni and G.C. Strinati, Phys. Rev. B 44, 7455 (1991).

17 Cf., e.g., A. Auerbach, Interacting Electrons and Quantum Magnetism (Springer-Verlag, New York, 1994 ), Chapt.3.

${ }^{18}$ It can be further verified that $\omega(\mathbf{q})$ given by Eq. (57) is symmetric under the separate transformations $\left(q_{x} \rightarrow q_{y}, q_{y} \rightarrow q_{x}\right)$ and $\mathbf{q} \rightarrow-\mathbf{q}$.

${ }^{19}$ Cf., e.g., N.W. Ashcroft and N.D. Mermin, Solid State Physics (Saunders College, Philadelphia, 1976), Chapt.32.

${ }^{20}$ R. Coldea, S. M. Hayden, G. Aeppli, T. G. Perring, C. D. Frost, T. E. Mason, S-W. Cheong, and Z. Fisk, preprint condmat/0006384

${ }^{21}$ B.R. Cooper, R.J. Elliott, S.J. Nettel, and H. Suhl, Phys. Rev. 127, 57 (1962).

${ }^{22}$ M.P. Gelfand, R.P. Singh, and D.A. Huse, Phys. Rev. B 40, 10801 (1989).

${ }^{23}$ D.C. Mattis, The Theory of Magnetism I (Springer-Verlag, New York, 1988), Sect. 6.6. 